\title{
Online Fault Detection and Isolation in Advanced Heavy Water Reactor using Multi-Scale Principal Component Analysis
}

\author{
Vidya Sagar Yellapu, Vineet Vajpayee, Akhilanand Pati Tiwari
}

\begin{abstract}
Signals from sensors placed at different locations are used for control and protection purposes in a nuclear reactor. It also requires in-service Fault Detection and Isolation (FDI) for its safe operation. The sensor signals are generally a superimposition of low-frequency components representative of true values of the variables being monitored; occasional high-frequency periodical oscillations due to disturbances and faults; and sensor faults. Techniques like Principal Component Analysis (PCA) can be used for FDI, however, it would be more meaningful if the FDI technique can also help for predictive maintenance of reactor internals through vibration spectra. To address these issues, a multi-scale PCA, integrating wavelet transform with PCA and aiming to reduce the modeling cost by using only a fewer scales that contribute to the monitoring has been proposed in this paper for online FDI of Advanced Heavy Water Reactor (AHWR), A new mathematical formulation of the Generalized Likelihood Ratio Test for its use with wavelet approximation coefficients has also been proposed for better sensor-FDI outcomes. The proposed approach detects and isolates sensor faults and process faults using the signals from neutron detectors. Efficacy of the proposed technique is established on the simulated ex-core ion chamber data of AHWR considering different scenarios that involve localized frequency contents representative of process faults, slowly developing (incipient) sensor faults, and the simultaneous presence of two or more of these scenarios. Simulation results validate the effectiveness of the proposed scheme for online FDI in the reactor.
\end{abstract}

Index Terms-Fault Detection and Isolation, Ex-core neutron detectors, Ion chambers, Advanced Heavy Water Reactor, Principal Component Analysis, Wavelets.

\section{INTRODUCTION}

$\mathbf{N}$ UCLEAR reactors being safety critical systems need multiple sensors for measurement of key parameters such as flow, pressure, temperature, and neutron flux. The sensor signals generally consist of both static and dynamic components representing slow as well as fast varying phenomena in the reactors. The static component corresponds to process variables measured by the sensors, whereas the dynamic component results from inherent fluctuations in process variables as a result of external disturbances on the plant such as random neutron flux, random heat transfer, turbulence, vibration, and

Vidya Sagar Yellapu is with EEE Department, SRKR Engineering College, Bhimavaram-534204, Andhra Pradesh, India. (e-mail: vidyasagar.yellapu@gmail.com).

Vineet Vajpayee is with School of Energy and Electronic Engineering, University of Portsmouth, Portsmouth, PO1 3DJ, UK (e-mail: vineet.vajpayee@port.ac.uk, vineet25iitr@gmail.com).

Akhilanand Pati Tiwari is with Bhabha Atomic Research Center, Mumbai 400085, India. He is also a Senior Professor in Homi Bhabha National Institute, Mumbai 400094, India (e-mail: aptiwari@barc.gov.in). other effects [1]. For instance, neutron detectors carry the signal corresponding not only to the neutron flux which is a slowly varying signal but also to the vibration of reactor internals. The hardware redundancy among sensors, which is always maintained for continuous safe-operation of the reactor even when some sensors malfunction, plays a vital role in Fault Detection and Isolation (FDI). Analytical redundancy or mathematical relationships among sensor signals can be found through either first principles approach or using statistical techniques like Principal Component Analysis (PCA) [2], [3]. PCA-based FDI, though proved to be highly efficient for sensor fault detection as presented in [3], acts only on the static components of the detector signals to find the possible degradation. However, extending PCA-based FDI to the process condition monitoring and diagnostics in addition to the sensor anomalies will be of more interest.

Generally, the neutron detector signals also contain measurement noises that may arise from different sources like stochastic nature of neutron interaction, fluctuation in pressure and temperature, mechanical vibration of internal parts, and so on. Neutron noise analysis can be used as a predictive maintenance strategy to safeguard the structural integrity, which would be otherwise at a risk due to vibration induced accidents. The neutron noises obtained from in-core or excore detectors, in the typical range of $0.5 \mathrm{~Hz}$ to $25 \mathrm{~Hz}$, give important information for in-service monitoring of the core for excessive mechanical motion of fuel assemblies, incore detector tubes, the reactor core support barrel, reactor vessel, and reactor coolant pumps [1], [4]-[8]. In literature, various works on the analysis of neutron detector noises using frequency domain techniques namely, fast Fourier transform, auto power spectral density, and cross power spectral density have been reported for different reactor configurations [4][10]. Though these frequency domain techniques were proved to be successful in detecting vibration-induced faults visible in the frequency spectrum, they ignore the cross-correlations among the static component of the sensor signals as they need to be applied individually on each signal. This limits their application, as the information hidden in cross-correlation is left unused, which plays a vital role in the detection of sensor faults. With the objective of detecting sensor faults, PCA which captures cross-correlations among the variables has been widely used in nuclear engineering [3], [11]-[13]. In most of the reported works, PCA models are developed for the underlying process assuming its existence only at a single scale in measurement space. However, it is well known that 
in systems like large nuclear reactors, multiple modes interact simultaneously and many time-frequency localized phenomena evolve at different scales of time [14]. These events may not be clearly visible at a single scale in measurement space. Thus, it is imperative to have data representation at appropriate scales or resolutions. Wavelets provide an architecture for multiscale data visualization due to their inherent multi-resolution approximation property and simultaneous time-frequency localization capability. They decompose noisy signals at different resolutions, thereby they extract features relevant to process dynamics and efficiently remove noise contribution. Processing based on wavelets minimizes distortion of signal bandwidth and thus improves the signal-to-noise ratio as compared to the frequency domain-based techniques which reduce only the high-frequency components of a signal and require that signal and noise spectra must be separable. Recently, the advantages of wavelets in processing neutron detector data, power transients, and in fault diagnosis have been reported [15]-[18].

In the last two decades, wavelet-based multi-scale techniques have demonstrated superior performance over the conventional single-scale approaches in different applications such as modeling, prediction, data-compression, and rectification. For process monitoring, Bakshi [19] proposed a Multi-Scale Principal Component Analysis (MSPCA) formulation where signals are decomposed at different scales using wavelet transform and then PCA is applied at individual scales to monitor the frequency induced signatures. This technique is very much suitable for industrial processes where non-stationary multiscale events are inherently existing. The approach simultaneously extracts auto-correlation within a sensor using wavelets and cross-correlation across the sensors through PCA. In [20] and [21], applications of MSPCA in FDI are demonstrated and it is shown that MSPCA outperforms the classical PCA technique. In most of the works, all the scales were utilized for building a PCA model. However, in practice, not all the scales contribute to process monitoring and control. For instance, usually the finest scale detail contains contribution arising mainly from high-frequency noise components. Hence, a significant reduction in the modeling effort can be achieved by selectively removing the detail scales not contributing much. For this, the occurrence of significant scales is to be found. In this work, the classical MSPCA method has been modified by identifying and building parsimonious models only at those scales where the underlying process evolves predominantly.

Therefore, the proposed work develops a novel online MSPCA-based FDI for process condition monitoring and diagnostics. Specifically, the technique is applied to the Advanced Heavy Water Reactor (AHWR) [22] in which coolant flowinduced vibrations of fuel may be caused [23]. This could be seen as a process fault that results in the variation of the frequency spectrum. Neutron noise signatures obtained from the ex-core ion chamber signals of AHWR are analyzed and statistics like Squared Prediction Error (SPE) are computed at significant scales to observe the on-set of vibration-induced faults. In addition, sensor faults or degradation can be identified from the measurements violating the algebraic relation- ships dictated by the constraint models obtained through PCA, when applied on either the measurement data or on the data selectively reconstructed after inverse-wavelet transformation or on the low-frequency approximation coefficients obtained through wavelets. FDI tools like Generalized Likelihood Ratio Test (GLRT) [24] integrated with MSPCA as used in [25], [26] for the detection of the process faults can also be used for dealing with the sensor faults. For this, fault signature matrices need to be constructed from the constraint residuals to identify the faulty sensor and the fault magnitude. However, none of the works in the literature used such an MSPCA-based GLRT formulation. In this paper, such formulation is used for sensorFDI based on the original measurement data and the data reconstructed with parsimonious MSPCA models discussed before. Further, a new mathematical formulation of GLRT is also proposed so that it can process the wavelet approximation coefficients for better sensor-FDI outcomes such as quick detection, accuracy in fault location and that in the estimate of the fault magnitude. The effectiveness of the proposed scheme is established for the case of an incipient fault in one of the ion chambers of AHWR.

The rest of the paper is organized as follows: Section II briefly discusses wavelets, PCA, and GLRT. Section III formulates the MSPCA scheme for FDI. Section IV demonstrates the application of the proposed technique to AHWR through the ex-core ion chambers. Section V presents the results of analysis when MSPCA is applied on the simulated ion chamber signals of AHWR. Finally, conclusions are drawn in Section VI. The fault signature matrices and the other formulations for GLRT based on approximation coefficients; and PCA models obtained from the original, reconstructed and the approximations data are respectively given in Appendix A and Appendix B.

\section{Wavelets, Principal Component AnAlysis, AND GENERALIZED LIKELIHOOD RATIO TEST}

\section{A. Wavelet Transform}

Wavelets are the atoms with variable time-frequency resolution for localization of the events. They are appropriate for analyzing phenomena having short-lived high-frequency components and long-lived low-frequency components or in which the former are superimposed on the latter [27], [28]. The Continuous Wavelet Transform (CWT) of a square integrable function $y(t)$ can be expressed as the correlation between $y(t)$ and the dilated and translated version of wavelet function. It is given by the inner product of $y(t)$ and the wavelet function as

$$
\begin{aligned}
W(s, \tau) & =\left\langle y(t), \frac{1}{\sqrt{s}} \psi\left(\frac{t-\tau}{s}\right)\right\rangle \\
& =\frac{1}{\sqrt{s}} \int_{-\infty}^{\infty} y(t) \psi^{*}\left(\frac{t-\tau}{s}\right) d t
\end{aligned}
$$

where ${ }^{*}$ represents the complex conjugate. The wavelet function $\psi$ integrates to zero and has finite energy [28]. The CWT operation maps a one dimensional function $y(t)$ to a function of two variables $s \in \mathbb{R}^{+}$and $\tau \in \mathbb{R}$ which respectively represent wavelet dilation and translation parameters. The CWT coefficients $W(s, \tau)$ give a redundant wavelet representation. However, a computationally efficient non-redundant 
representation can be obtained by sampling $s$ and $\tau$ on a dyadic grid known as the Discrete Wavelet Transform (DWT). The DWT coefficients are determined by selecting $s=2^{j}$ and $\tau=2^{j} l$, where $j$ and $l$ are scale and position indices respectively with $j, l \in \mathbb{Z}$, as given by

$$
W\left(2^{j}, 2^{j} l\right)=\frac{1}{\sqrt{2^{j}}} \int_{-\infty}^{+\infty} y(t) \psi^{*}\left(\frac{t-2^{j} l}{2^{j}}\right) d t
$$

Therefore, using (2), function $y(t)$ can be represented as

$$
\begin{aligned}
y(t) & =\sum_{j=-\infty}^{+\infty} \sum_{l=-\infty}^{+\infty} a(j, l) 2^{-j / 2} \psi\left(2^{-j} t-l\right) \\
& =\sum_{j=-\infty}^{+\infty} \sum_{l=-\infty}^{+\infty} a(j, l) \psi_{j, l}(t)
\end{aligned}
$$

where the two-dimensional sequence $a(j, l)$ constitutes wavelet coefficients. Equation (3) indicates the multiresolution ability of the wavelet functions. It constructs a hierarchy of approximations in various nested subspaces. The sub-spaces are defined by scaling function, $\phi(t)$ and wavelet function, $\psi(t)$, which carry the signal decomposition in approximation and detail sub-spaces respectively. Thus, from (3),

$$
y(t)=\sum_{l=-\infty}^{\infty} c_{J+1, l} \phi_{J+1, l}(t)+\sum_{j=0}^{J} \sum_{l=-\infty}^{\infty} d_{j, l} \psi_{j, l}(t),
$$

where the coefficients $c_{J+1, l}$ and $d_{j, l}$ are called as approximation and detail coefficients respectively. Scale $J$ represent the maximum depth of decomposition while $J+1$ represents approximation at $J^{\text {th }}$ scale.

\section{B. Principal Component Analysis}

The real-time data as collected on the ion chamber signals of a reactor have the stochastic properties along with the deterministic nature as given by the $n$-variate noisy data vector $\boldsymbol{y}=\boldsymbol{x}+\boldsymbol{\varepsilon}$, which is the sum of the true data vector $\boldsymbol{x} \in \mathbb{R}^{n}$ and the white noise vector $\varepsilon \in \mathbb{R}^{n}$. Assuming that $m$ number of variables $(m<n)$ in $\boldsymbol{x}$ are dependent on other variables, there are $(n-m)$ significant eigenvalues whose corresponding eigenvectors span the principal component subspace and there are $m$ number of insignificant but non-zero eigenvalues that span the residual subspace. PCA projects the $n \times N$ dimensional data matrix $\boldsymbol{Y}=\left[\begin{array}{ll}\boldsymbol{y}_{1} & \boldsymbol{y}_{2} \ldots \boldsymbol{y}_{N}\end{array}\right]$ onto the subspaces with major and minor variabilities, where $\boldsymbol{y}_{k} \in \mathbb{R}^{n}, \forall k=1,2, \ldots, N$ represents the data vector at an instant $k$. To state it otherwise, the eigenvector matrix $\boldsymbol{V}=[\boldsymbol{P} \boldsymbol{B}]$, where $\boldsymbol{P}=\left[\begin{array}{lll}\boldsymbol{v}_{1} & \boldsymbol{v}_{2} \ldots \boldsymbol{v}_{n-m}\end{array}\right]$ represents the principal component subspace and $\boldsymbol{B}=\left[\begin{array}{ll}\boldsymbol{v}_{n-m+1} & \boldsymbol{v}_{n-m+2} \ldots \boldsymbol{v}_{n}\end{array}\right]$ represents the residual subspace and converts the correlated variables in $\boldsymbol{x}$ into uncorrelated scores given by

$$
\boldsymbol{t}_{i}=\boldsymbol{v}_{i}^{T} \boldsymbol{Y}, \forall i=1, \ldots, n
$$

for each of the eigenvectors $\boldsymbol{v}_{i}$ and thus separates $\boldsymbol{Y}$ into deterministic variation (due to change in process) and stochastic variation (due to noise). However, such projection requires the order of the residual subspace $m$, called the model order. Once it is known, we can decompose the sample data vector at an instant $k$ into the signal matrix $\hat{\boldsymbol{x}}_{k}$ and the noise matrix $\hat{\boldsymbol{e}}_{k}$ with the help of matrices $\boldsymbol{P}$ and $\boldsymbol{B}$ as follows:

$$
\boldsymbol{y}_{k}=\boldsymbol{P} \boldsymbol{P}^{T} \boldsymbol{y}_{k}+\boldsymbol{B} \boldsymbol{B}^{T} \boldsymbol{y}_{k}=\hat{\boldsymbol{x}}_{k}+\hat{\boldsymbol{e}}_{k} .
$$

The vector $\hat{\boldsymbol{x}}_{k}=\boldsymbol{P} \boldsymbol{P}^{T} \boldsymbol{y}_{k}$ gives the data vector reconstructed using the $n-m$ number of principal components in $\boldsymbol{P}$ and $\hat{\boldsymbol{e}}_{k}=\boldsymbol{B} \boldsymbol{B}^{T} \boldsymbol{y}_{k}$ gives the error in this reconstruction. The constraint residuals vector at time $k$ is given by

$$
\boldsymbol{r}_{k}=\boldsymbol{B}^{T} \boldsymbol{y}_{k}, \in \mathbb{R}^{m}
$$

and it has a mean value of $\mathbf{0}$, i.e., $\mathrm{E}\left[\boldsymbol{r}_{k}\right] \simeq \mathbf{0}, \forall i=1,2, \ldots, N$. Since the operation $\boldsymbol{B}^{T} \boldsymbol{y}_{k}=\boldsymbol{r}_{k} \simeq \mathbf{0}$ resembles the relation $\boldsymbol{A} \boldsymbol{x}=\mathbf{0}$ on an average sense, $\boldsymbol{B}^{T}$ is taken equivalent to the constraint model $\boldsymbol{A}$ that shows the relationships among the variables in the measurement vector $\boldsymbol{y}$ [3].

At any instant $k$, SPE or $Q$ statistics [13], [20] to take decisions about the sensor faults can be computed as

$$
Q_{k}=\boldsymbol{y}_{k}^{T}\left(\boldsymbol{I}-\boldsymbol{P} \boldsymbol{P}^{T}\right) \boldsymbol{y}_{k} .
$$

For a false alarm probability $\alpha$, the threshold value of $Q$ statistic can be computed as [25]

$$
Q_{\alpha}=g \chi_{\alpha, h}^{2},
$$

where $g=\frac{\operatorname{variance}(Q)}{2 \times \text { mean }(Q)}$ and $\chi_{\alpha, h}^{2}$ is the value of chi-square distribution at a significance level of $\alpha$ with degrees of freedom given by $h=\frac{2 \times \text { mean }(Q)^{2}}{\text { variance }(Q)}$. In online FDI, if the SPE statistic $Q_{k}$ computed from (8) exceeds the threshold $Q_{\alpha}$, a fault is declared and the source and magnitude of fault can be investigated through the techniques such as GLRT.

\section{Generalized Likelihood Ratio Test}

In GLRT, the fault signature vectors $\boldsymbol{f}_{j}=\boldsymbol{A} \boldsymbol{e}_{j}$ are developed for each measurement $j$, where $\boldsymbol{e}_{j}$ is the unit vector with 1 at position $j$. The fault signature matrix that holds fault signature vectors as its columns can be expressed as [24]

$$
\boldsymbol{F}_{i}=\left\{\begin{array}{l}
\boldsymbol{A} \boldsymbol{e}_{i_{1}}, i_{1}=1, \ldots, n ; \\
\boldsymbol{A}\left(\boldsymbol{e}_{i_{1}}, \boldsymbol{e}_{i_{2}}\right), \forall i_{1}, i_{2}=1, \ldots, n, i_{1} \neq i_{2} ; \ldots ; \\
\boldsymbol{A}\left(\boldsymbol{e}_{i_{1}}, \boldsymbol{e}_{i_{2}}, \ldots, \boldsymbol{e}_{i_{g}}\right), \forall i_{1}, i_{2}, \ldots, i_{g}=1, \ldots, n, \\
\quad i_{1} \neq i_{2} \neq \ldots \neq i_{g},
\end{array}\right.
$$

where the subscript $i$ refers to the set of combinations in which $i_{1}, i_{2}, \ldots, i_{g}$ are chosen to exhaustively consider all possible combinations of number of simultaneous sensor-faults from $1,2, \ldots, g$. The residuals have a mean of $\mathbf{0}$ and $\boldsymbol{F}_{i} \boldsymbol{b}_{k}$ in the presence of no and $g$ faults hypothesized respectively, where $\boldsymbol{b}_{k}$ is a column vector of unknown magnitudes of faults at the instant $k$.

If $p($.$) denotes the probability density function, then the$ Generalized Likelihood Ratio of the $m$-variate residuals obtained from (7) can be written as

$$
\lambda(\boldsymbol{r})=\sup \frac{p\left(\boldsymbol{r} \mid H_{1}\right)}{p\left(\boldsymbol{r} \mid H_{0}\right)},
$$


where $H_{1}$ and $H_{0}$ respectively represent the hypotheses of fault and no-fault. Using the normal probability density function for the residuals $\boldsymbol{r}$, (11) can be written as

$$
\lambda(\boldsymbol{r})=\sup _{i, \boldsymbol{b}_{k}} \frac{\exp \left\{-\frac{1}{2} \boldsymbol{\varrho}_{i, k}^{T} \boldsymbol{\Sigma}_{r}^{-1} \boldsymbol{\varrho}_{i, k}\right\}}{\exp \left\{-\frac{1}{2} \boldsymbol{r}_{k}^{T} \boldsymbol{\Sigma}_{r}^{-1} \boldsymbol{r}_{k}\right\}},
$$

where $\varrho_{i, k}=\boldsymbol{r}_{k}-\boldsymbol{F}_{i} \boldsymbol{b}_{k}$. Defining $L=2 \ln \lambda(\boldsymbol{r})=\sup _{i} L_{i, k}$, where

$$
L_{i, k}=\boldsymbol{r}_{k}^{T} \boldsymbol{\Sigma}_{r}^{-1} \boldsymbol{r}_{k}-\sup _{\boldsymbol{b}_{k}} \boldsymbol{\varrho}_{i, k}^{T} \boldsymbol{\Sigma}_{r}^{-1} \varrho_{i, k},
$$

the maximum likelihood estimates of the fault magnitudes $\hat{\boldsymbol{b}}_{k}$ is obtained by equating the first derivative of (13) with respect to $\boldsymbol{b}_{k}$ to zero, and is

$$
\hat{\boldsymbol{b}}_{k}=\left(\boldsymbol{F}_{i}^{T} \boldsymbol{\Sigma}_{r}^{-1} \boldsymbol{F}_{i}\right)^{-1}\left(\boldsymbol{F}_{i}^{T} \boldsymbol{\Sigma}_{r}^{-1} \boldsymbol{r}_{k}\right),
$$

and the corresponding test statistics are

$$
L_{i, k}=\left(\boldsymbol{F}_{i}^{T} \boldsymbol{\Sigma}_{r}^{-1} \boldsymbol{r}_{k}\right)^{T}\left(\boldsymbol{F}_{i}^{T} \boldsymbol{\Sigma}_{r}^{-1} \boldsymbol{F}_{i}\right)^{-1}\left(\boldsymbol{F}_{i}^{T} \boldsymbol{\Sigma}_{r}^{-1} \boldsymbol{r}_{k}\right) .
$$

The false alarm probabilities for each of the test statistics $L_{i, k}$ is

$$
\alpha_{i, k}=p\left(\chi_{g, \alpha}^{2} \geq L_{i, k}\right)
$$

where $\chi_{g, \alpha}^{2}$ is a random variable following a chi-square distribution with $g$ degrees of freedom. The combination $i$ chosen out of $\left\{i_{1}=1, \ldots, n ; i_{1}, i_{2}=1, \ldots, n, i_{1} \neq\right.$ $\left.i_{2} ; \ldots ; i_{1}, i_{2}, \ldots, i_{g}=1, \ldots, n, i_{1} \neq i_{2} \neq \ldots \neq i_{g}\right\}$ and corresponding to minimum false alarm probability gives the number and locations of faults and the bias magnitudes.

For the evaluation of GLRT, the following indices can be computed with the help of the hypothesis testing in which the null hypothesis $H_{0}$ states that no fault is present; and the alternative hypothesis $H_{1}$ states that faults are present in one or more signals [3]:

1) Overall Detection Rate $(O D R)$ : It is the percentage of detection of one or more faults or rejection of $H_{0}$ out of total number of trials. It involves detection, even during the cases where $H_{0}$ is true. For a true $H_{1}$, ODR is desirable to be close to $100 \%$ [3].

2) Overall Power $(O P)$ : It is the percentage of trials when one or more faults are correctly identified for a true $H_{1}$. For a true $H_{1}$, OP should be close to $100 \%$ [3].

3) Mean-Square Error (MSE): It is a measure of error in the estimate of fault magnitude in a sensor, defined as

$$
\sqrt{\frac{\sum_{k \in C}\left(\boldsymbol{b}_{k}-\hat{\boldsymbol{b}}_{k}\right)^{T}\left(\boldsymbol{b}_{k}-\hat{\boldsymbol{b}}_{k}\right)}{N_{C}}},
$$

where $C$ is the set of instants at which the faults are correctly identified and $N_{C}$ is the cardinality of $C$ [29].

\section{Multi-ScAle Principal COMPONENT ANALYSiS FOR ON-LINE FDI}

MSPCA for FDI involves different sequential operations on the multi-variate data, namely decomposition of the data into multiple scales using wavelets, the projection of this decomposed data onto the principal components, and inverse wavelet decomposition for reconstructing the data. $Q$ statistics are computed from the appropriate variables from (9), which will be used for MSPCA-based FDI whose objectives are the detection of vibration signatures of process internals and sensor faults. These objectives are met by a one-time executed off-line algorithm for model development and a continuously running online algorithm for every new observation [21]. The concepts involved in the MSPCA-based FDI are discussed in this section.

\section{A. Multi-scale Data Decomposition}

For a given time-series $\overline{\boldsymbol{y}} \in \mathbb{R}^{2^{J} \times 1}$, the DWT operation in terms of matrix multiplication is given by

$$
\boldsymbol{y}^{w}=\left[\begin{array}{lllll}
\boldsymbol{y}_{J+1}^{T} & \boldsymbol{y}_{J}^{T} & \boldsymbol{y}_{J-1}^{T} & \cdots & \boldsymbol{y}_{1}^{T}
\end{array}\right]^{T}=\boldsymbol{W} \overline{\boldsymbol{y}},
$$

where $\boldsymbol{y}^{w}$ is called as wavelet coefficient and comprised of approximation (at scale $J+1$ ) and details (at scales $j=1,2, \ldots, J)$. The wavelet operator $\boldsymbol{W}$ is given by

$$
\begin{aligned}
\boldsymbol{W} & =\left[\begin{array}{llllll}
\prod_{j=1}^{J} \boldsymbol{H}_{j}^{T} & \boldsymbol{G}_{J}^{T_{j=1}^{J-1} \boldsymbol{H}_{j}^{T}} & \boldsymbol{G}_{J-1}^{T} \prod_{j=1}^{J-2} \boldsymbol{H}_{j}^{T} & \cdots & \boldsymbol{G}_{1}^{T}
\end{array}\right]^{T}, \\
& =\left[\begin{array}{lllll}
\tilde{\boldsymbol{H}}_{J}^{T} & \tilde{\boldsymbol{G}}_{J}^{T} & \tilde{\boldsymbol{G}}_{J-1}^{T} & \cdots & \tilde{\boldsymbol{G}}_{1}^{T}
\end{array}\right]^{T}
\end{aligned}
$$

where $\tilde{\boldsymbol{H}}_{J}\left(1 \times 2^{J}\right)$ and $\tilde{\boldsymbol{G}}_{J}\left(2^{J-j} \times 2^{J}\right)$ are matrices of wavelet filter coefficients.

The effective implementation of wavelet-based techniques requires careful selection of wavelet and the maximum depth of decomposition. Generally, complex wavelets can be used for capturing oscillatory behaviour whereas real ones can be used for detecting peaks. For instance, real wavelets such as Haar are suitable for representing highly localized events like singularities or sudden changes due to their piecewise constant representation. They compute the transformation entirely using the past and present data; avoid undesirable border distortions due to signal extensions; and do not introduce any delay in the analysis [30]. Hence, Haar wavelets are chosen in the proposed scheme. The maximum depth or scale of decomposition $J$ is selected so as to ensure that a minimum number of observations hit the support of basis function. It can be found out using the Fourier transform such that the magnitude of the Fourier transform is above the noise floor-level [14], i.e., $\left|\boldsymbol{y}\left(\pi / 2^{J}\right)\right| \geq|\boldsymbol{y}(\pi / f)|$. It has been observed that not all the scales contribute in determining process behavior, thus for a parsimonious model representation, the selection of significant scales is a crucial step [14], [30].

The online implementation of wavelet transform for $J=2$ is shown in Fig 1. The signal is measured at finest scale $j=0$ and assumed to be available up to the current time instant $k$. A window of $2^{J}$ observations is formed and decomposed into detail spaces at $j=1,2, \ldots, J$ and an approximation space at $j=J+1$. With the availability of new data at every subsequent instant, the window is translated by one time step and the wavelet decomposition is performed. It may be noted that due to redundancy in representation, only the last (or rightmost) coefficients indexed as $k, k+1, \ldots$ at each scale are stored. 


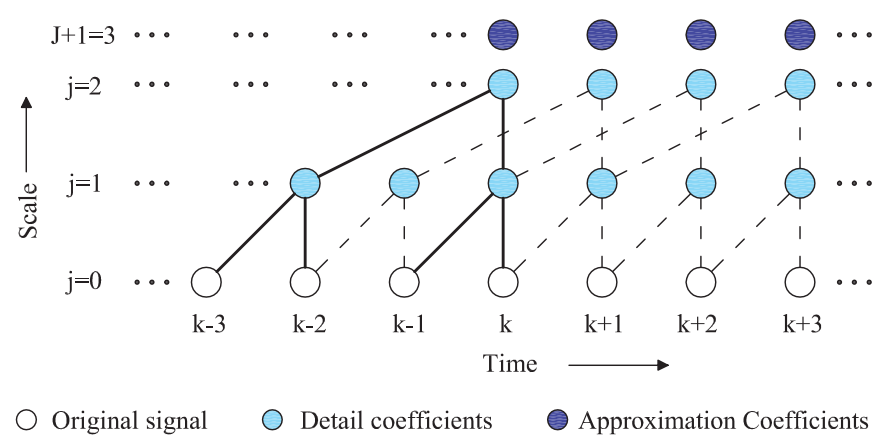

Fig. 1. Implementation of wavelet transform for $J=2$.

\section{B. Local PCA Models at Different Scales}

Once a decomposition depth $J$ is worked out, PCA is applied to each of the $J+1$ matrices ( $J$ matrices holding details and 1 matrix holding approximation coefficients) with an objective of extracting cross-correlation of the sensors. However, the challenge lies in choosing the model order $m_{j}, j=1,2, \ldots, J+1$ at each scale accurately. There are many methods for determining model order [2], out of which the method based on Cumulative Percentage of Variance (CPV) of principal components is used here. According to this method, the minimum number of principal components, the cumulative sum of whose eigenvalues exceeds $95 \%$ of the sum of all eigenvalues is taken as $p_{j}$, the order of principal component subspace. Thus, the model order is obtained as the order of the subspace of insignificant variations as $m_{j}=n-p_{j}$. It should be noted that the data at each scale may have different values for $m_{j}$ that decides the dimensions of the matrices $\boldsymbol{P}_{j}$ and $\boldsymbol{B}_{j}$ of that scale.

If the frequency spectrum of the process is changed with an addition of one or more frequency components as a result of faults, the $Q_{j}$ statistics at the scales corresponding to added frequencies show large values due to broken covariance structure of the details-data while their values for rest of the frequency spectrum, remain by and large unaffected. Hence, these statistics can be continuously monitored for the detection and identification of process faults.

Moreover, sensor faults can be detected with the help of $Q$ statistics computed from the approximations obtained in the wavelet transformation. As mentioned before, a new mathematical formulation has been suggested to use GLRT on the wavelet approximation coefficients.

Lemma III.1. When the GLRT is applied on the wavelet approximation coefficients, the fault signature matrix in various hypothesized-number of sensor faults up to $g$ is

$\boldsymbol{F}_{i}=\left\{\begin{aligned} \boldsymbol{A}_{A} \boldsymbol{E}_{i_{1}} \tilde{\boldsymbol{H}}_{J}^{T}, i_{1}=1, \ldots, n ; \\ \boldsymbol{A}_{A}\left(\boldsymbol{E}_{i_{1}} \tilde{\boldsymbol{H}}_{J}^{T}, \boldsymbol{E}_{i_{2}} \tilde{\boldsymbol{H}}_{J}^{T}\right), \forall i_{1}, i_{2}=1, \ldots, n, i_{1} \neq i_{2} ; \ldots ; \\ \boldsymbol{A}_{A}\left(\boldsymbol{E}_{i_{1}} \tilde{\boldsymbol{H}}_{J}^{T}, \boldsymbol{E}_{i_{2}} \tilde{\boldsymbol{H}}_{J}^{T}, \ldots, \boldsymbol{E}_{i_{g}} \tilde{\boldsymbol{H}}_{J}^{T}\right), \\ \quad \forall i_{1}, i_{2}, \ldots, i_{g}=1, \ldots, n, i_{1} \neq i_{2} \neq \ldots \neq i_{g},\end{aligned}\right.$

where $\boldsymbol{A}_{A}$ is the PCA-based constraint model obtained from approximation coefficients and $\boldsymbol{E}_{i} \in \mathbb{R}^{n \times 2^{J}}$ is a matrix whose $i^{\text {th }}$ row has its all elements equal to 1 .
Proof. Refer to Appendix A.

\section{Data Pre-processing and Synthesis}

Wavelet thresholding followed by signal reconstruction is a well-established denoised technique that enables nonlinear approximation of a signal [27]. Wavelet transform distributes contribution of noise among all small coefficients while contribution from signal remains in a small number of high amplitude coefficients. Thus, it decorrelates wavelet coefficients of the signal from that of the noise thereby preserving only relevant signal components. Thresholding operation can either be hard thresholding or soft thresholding. In case of hard thresholding, the coefficients below a certain threshold are made equal to zero, while in soft thresholding the coefficients are reduced by the given threshold. Wavelet pre-processing can also be performed by removing the entire contribution arising from insignificant details. The signal is then reconstructed only from the information present at significant scales.

The inverse wavelet transform to obtain the reconstructed signal in measurement domain is given by

$$
\hat{\boldsymbol{y}}=\overline{\boldsymbol{W}} \boldsymbol{y}^{w},
$$

where $\overline{\boldsymbol{W}}$ represent the inverse wavelet operator. $\boldsymbol{y}^{w}$ and $\hat{\boldsymbol{y}}$ contain pre-processed wavelet coefficients and reconstructed processed signal respectively.

\section{Off-line MSPCA algorithm}

The detailed sequence of operations to be performed for developing the process-history-based models are given in the following off-line algorithm:

1) Obtain the $n$-variate observations in sufficiently large number $N$ during a fault-free operation. Compute the level $J$ of wavelet decomposition as described in Sec. III-A and form the wavelet decomposition matrix $\boldsymbol{W}$

2) Decompose each of the $n$ variables in the data matrix $\boldsymbol{Y}$ into $J$ vectors of detail coefficients and 1 vector of approximation coefficients using $\boldsymbol{W}$.

3) Group the detail coefficients at each level of all variables as a matrix and approximation coefficients as another matrix, i.e., $J$ number of detail matrices and 1 number of approximation matrix.

4) Apply SVD on the data in each of $J+1$ matrices obtained in Step-3. For each of the $J+1$ data sets, choose the value of $m_{j}$ as explained in Sec. III-B and develop PCA models ( $\boldsymbol{P}_{j}$ and $\boldsymbol{B}_{j}$ matrices) from the eigenvectors of the data.

5) Compute $Q_{j}$ statistics using the PCA models at each scale and determine the thresholds $Q_{\alpha, j}$.

6) Perform data pre-processing and then apply inverse wavelet transformation on the processed data of each variable using $\bar{W}$.

This completes the development of process-history-based models which can now be used on the new observations.

\section{E. On-line MSPCA algorithm}

Once the off-line algorithm is executed, another algorithm has to be run which is similar to the off-line one but excludes 
the need for the repeated development of PCA models and wavelet transformation matrix $\boldsymbol{W}$. The data used at the instant $k$, denoted by $\boldsymbol{Y}_{k} \in \mathbb{R}^{n \times 2^{J}}$ comprise of all the $2^{J}$ instants prior to and including $k$. The steps involved are as follows:

1) Set observation index $k=1$. Load the data $\boldsymbol{Y}_{k}$, all the PCA models ( $\boldsymbol{P}_{j}$ and $\boldsymbol{B}_{j}$ matrices) developed for details and approximations; and wavelet transformation matrix $\boldsymbol{W}$.

2) Decompose $\boldsymbol{Y}_{k}$ into detail and approximation coefficients using the matrix $\boldsymbol{W}$.

3) Use PCA models on these coefficients to get $Q_{j}$ statistics $\forall j=1,2, \ldots, J+1$.

4) For all details with scale index $j=1,2, \ldots, J$, declare a process fault, if the $Q_{j}$ statistics exceed the corresponding thresholds $Q_{\alpha, j}$, and investigate the type of fault from the wavelet coefficients.

5) From the approximations, declare a sensor fault if $Q_{J+1}$ statistic exceeds the thresholds $Q_{\alpha, J+1}$, apply GLRT and output the FDI outcomes.

6) Selectively reconstruct the measurement data using inverse wavelet transformation matrix $\overline{\boldsymbol{W}}$ for their further use.

7) Increment $k$ by 1 , input new data $\boldsymbol{Y}_{k}$ and go to step-2.

The flow-chart representation of this online algorithm is given in Fig. 2.

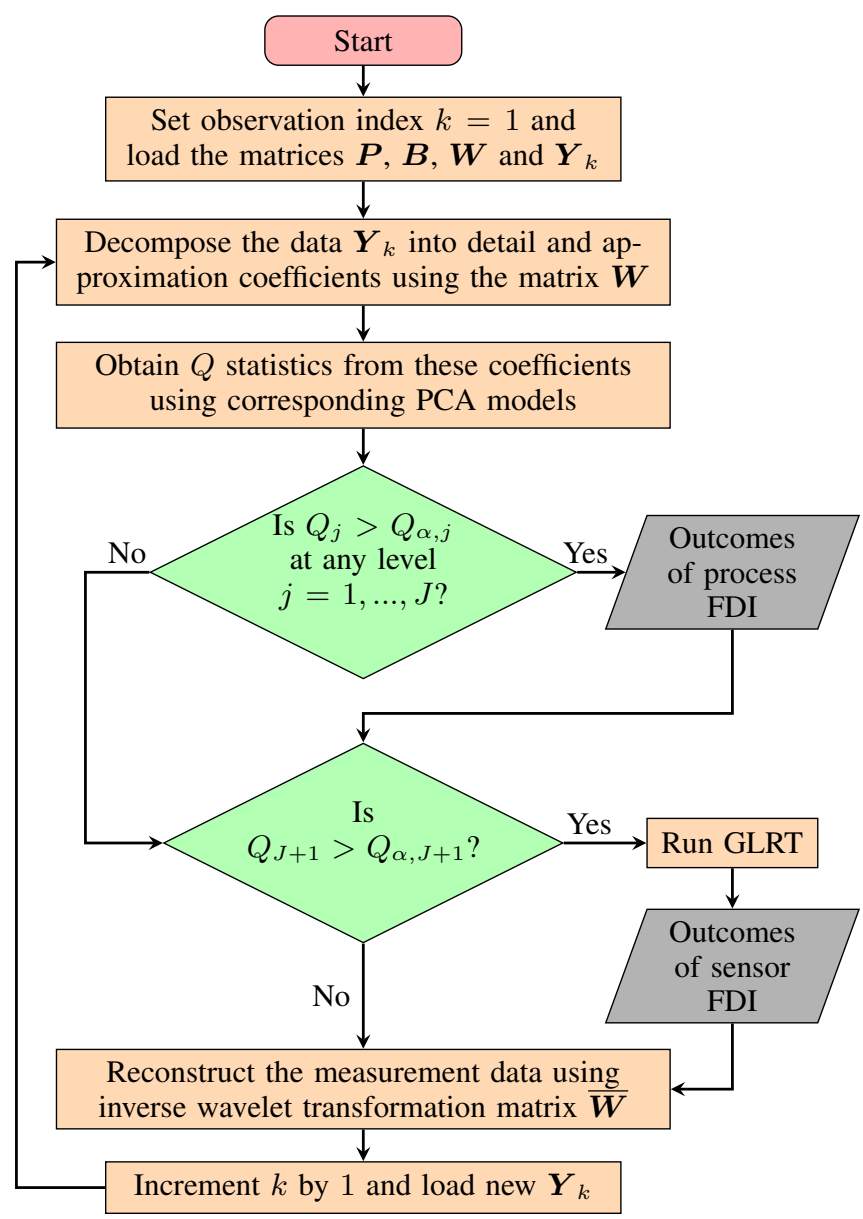

Fig. 2. Flow chart of MSPCA-based online FDI scheme

\section{ApPlicAtion of MSPCA-BASED FDI to ION CHAMBERS OF AHWR}

The MSPCA-based FDI scheme is applied to the ion chamber signals of AHWR, a 920 MW (thermal), vertical, pressure tube type, heavy-water moderated, boiling light-water cooled, natural circulation type reactor [22]. AHWR has 9 excore ion chambers located in the vault water surrounding the reflector [3], [31]. The ion chambers sense the leakage flux entering into the vault water, which in turn is proportional to the core-average neutron flux. Out of the 9 ion chambers, 3 ion chambers (namely ion chamber-1, 2, 3) are meant for reactor control and monitoring, 3 ion chambers (namely ion chamber$4,5,6)$ are for primary shut-down system denoted as ShutDown System-1, and remaining 3 (namely ion chamber-7, 8, 9) are for secondary shut-down system denoted as Shut-Down System-2.

Like any other large reactor, AHWR is also prone to vibrations of internal parts. For instance, a high-velocity coolant flow can excite fuel bundle and fuel elements inside it thereby resulting in vibrations of the fuel bundle. In AHWR, the vibration spectrum, in this case, is found out to have two cluster modes around 5 and $11 \mathrm{~Hz}$ [23]. These low-frequency vibrations may cause inter-element rubbing that can eventually damage the fuel bundle. There are other possible ways also that lead to vibration-induced damages. In all the significant scenarios leading to vibrations, the frequencies of vibrations are less than $30 \mathrm{~Hz}$ [1], [4]-[10]. As these are low frequencies, their resolution with accelerometers, which are suitable for high frequencies, is a complex phenomenon. However, the resolution is quite easy with ion chamber signals as they are sensitive to neutron flux distribution, which is affected due to the vibrations of the internal parts of the reactor. For example, thermo-hydraulic fluctuations generate oscillatory behaviour in the ion chamber signals at around $1 \mathrm{~Hz}$, whereas the real value of the core average flux continues to exhibit its normal behaviour. The second mode of oscillation is also generally present in some of the process-faults like the vibration of the deck plate as in the case of fuel assembly vibrations.

Taking different aspects into consideration, the current from the linear amplifier of an ion chamber- $l$ can be represented as

$$
i_{l}=K \phi_{l}+\varepsilon_{l}+\mu+b_{l}+4 \mathrm{~mA}, l=1,2, \cdots, 9,
$$

where $K=10.667$ is the product of detector sensitivity and the gain of the amplifier stages and $\phi_{l}$ denotes the per-unit (p.u.) value of the local neutron flux at the $l^{\text {th }}$ ion chamber location, which is directly proportional to the core average flux for nominal flux distribution. $\varepsilon_{l}$ is the measurement noise component in the uncertainty vector $\varepsilon$ as discussed in Section II-B. The noise $\varepsilon$ is generally independent and normally distributed with the following properties [32]:

$$
E[\varepsilon]=\mathbf{0}, \quad E\left[\varepsilon_{k} \varepsilon_{l}^{T}\right]=\boldsymbol{R} \delta_{k, l},
$$

where $\boldsymbol{R}$ is the covariance matrix of the measurement uncertainties, and $\delta_{k, l}$ is the Kronecker delta. The parameter $\mu$ in (20) denotes sinusoidal functions representative of process 
faults, if any, that induce vibrations as given in

$$
\mu=\sum_{k=1}^{h} M_{k} \sin \left(2 \pi f_{k} t\right),
$$

where $M_{k}$ and $f_{k}$ are the magnitude and frequency of the $k^{\text {th }}$ frequency component respectively. Lastly, $b_{l}$ is the degradation in the $l^{\text {th }}$ ion chamber signal due to a sensor fault, if any.

The frequency dependent noise signatures in the ion chamber currents, approximated by (20), are measured regularly for process-fault detection [1], [4]-[8]. After the detection of process faults, the sources of noise components can be identified by comparing the measured noise signatures with baseline signatures of each anticipated fault. MSPCA-based scheme for FDI can then be designed so as to capture one or more of the noise signatures at different scales. The $Q$ statistics computed at these scales play a major role in discriminating between different faults. It may also be noted that though $b_{l}$ may represent either an incipient fault or an abrupt fault in the sensor, its time-dependent magnitude is immaterial for the analysis like PCA-based FDI that are concerned only about cross-correlation in the ion chamber signals but not the auto-correlation. The $Q$ statistics can be computed on the reconstructed measurement data for fault detection, which is followed by fault identification with GLRT.

Fig. 3 depicts the implementation of the proposed MSPCAbased scheme for FDI in AHWR. Each of the control and protection systems is fed with signals from linear amplifiers of the ion chambers. Although signals are tapped from the 9 ion chambers before being fed to the MSPCA-based FDI system, physical separation and electrical isolation among control and each of the protection systems are maintained. The time series data of all 9 ion chambers in steady-state and in transient situations simulated using a mathematical model of the AHWR core and the ion chamber model given by (20), as described in [31], is supplied to the online MSPCA-based scheme for FDI as shown in Fig. 3. The wavelet transform block decomposes the data from each ion chamber into approximation and details based on the decomposition depth $J$. PCA is applied on the approximation and details at each level of all the 9 ion chamber data. The violations of $Q$ statistics at each level $j=1,2, \ldots, J$ are checked against the corresponding thresholds to detect the process faults, if any. On the other hand, the violations of $Q$ statistics at level $J+1$ are checked against the threshold to detect the sensor faults, if any. If sensor faults are detected, GLRT is applied to know the faulty sensor index and the fault estimate. After the FDI is performed, the inverse wavelet transformation is applied to the pre-processed data to get the data in measurement space for their further use by control and protection systems.

\section{RESUlts And AnAlysis}

In this section, a detailed analysis is performed on the results obtained through MSPCA approach on the ion chamber data simulated through the mathematical model of AHWR. A demand power change transient is considered, wherein the demand power change from 1.0 p.u. to 0.9 p.u. is reflected in all the 9 ion chamber signals. The data of ion chamber signals is simulated for $140 \mathrm{~s}$ out of which the reactor is at steady-state producing 1.0 p.u. till $100 \mathrm{~s}$ at which it undergoes the demand power change transient. A sampling time of $0.02 \mathrm{~s}$ leads to the generation of 7000 observations on 9 ion chambers. The measurement noise in (20) is assumed to be normally distributed with a standard deviation of $0.29 \mathrm{~mA}$, which is equivalent to $2 \%$ random fluctuations around the full power steady-state.

Simulations are performed in such a way that one simulation is sufficient to cover different scenarios. The scenarios considered involve the data carrying no fault, one or two frequencies indicative of process faults, one frequency along with a sensor fault, two frequencies along with a sensor fault, a sensor fault alone, process change and sensor fault together, one or two frequency components with simultaneous presence of both process change and sensor fault. To include all these scenarios in a single simulation, the data of 7000 observations length is divided into different windows numbered from I to XIII as shown in Fig. 4. The scenarios in each of these windows are as follows:

1) Window-I: The data in this window are used for the computation of $J, \boldsymbol{W}, \boldsymbol{P}$, and $\boldsymbol{B}$.

2) Window-II: The data in this window are considered to be fault-free, i.e., there are no process-fault induced frequencies, no sensor fault, and there is no process change as well. This case is considered to test the algorithm against the no-fault case.

3) Window-III: In this window, the data carry components of a single frequency, say $f_{1}$.

4) Window-IV: In this window, the data carry components of a single frequency, say $f_{2} \neq f_{1}$.

5) Window-V: This data window is corrupted by two different frequency components $f_{1}$ and $f_{2}$.

6) Window-VI: In this window, one of the sensors, namely ion chamber-5, is assumed to start developing an incipient fault along with the presence of fault-induced frequency $f_{1}$. The rate of rise of this incipient nature is assumed to be constant at $0.2 \%$ of the nominal value of the signal at steady-state $(14.67 \mathrm{~A})$ per second such that the fault shows the nature of a ramp signal. It is also assumed that the rate of rise of fault magnitude persists in other subsequent windows as well such that the fault grows by a value of $2.934 \mathrm{~A}$ for every $100 \mathrm{~s}$. The growth of this sensor-fault magnitude is as shown in Fig. 5.

7) Window-VII: In this window, ion chamber-5 is assumed to be faulty as described in point- 6 above, along with the presence of fault-induced frequency $f_{2}$.

8) Window-VIII: This data window has both fault-induced frequencies $f_{1}$ and $f_{2}$ along with a sensor fault in ion chamber-5.

9) Window-IX: In this window, only the incipient fault in the ion chamber-5 is considered.

10) Window-X: This window constitutes both the sensor fault in ion chamber-5 along with the commencement of process change from 1.0 p.u. and its way towards 0.9 p.u.

11) Window-XI: This data window holds the frequency $f_{1}$, sensor fault in ion chamber-5 and the process change that 


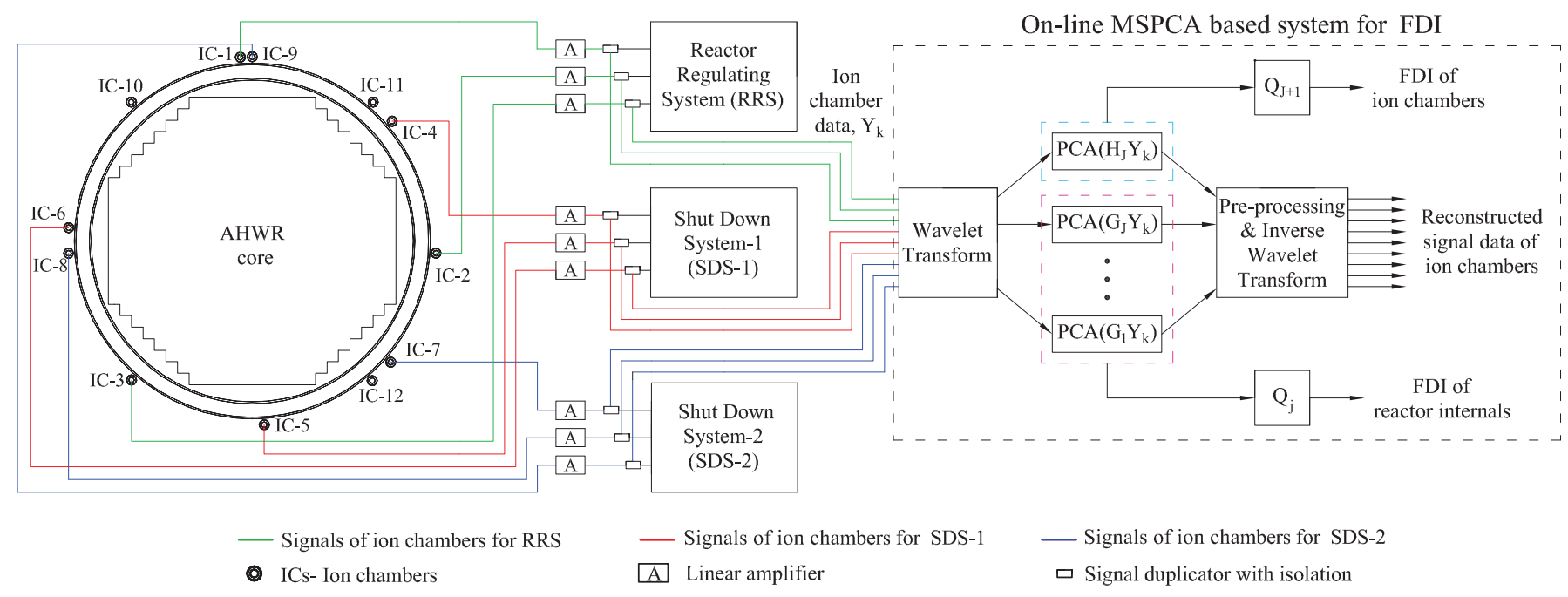

Fig. 3. Implementation of the proposed MSPCA-based method for FDI in AHWR.

commenced in Window-X.

12) Window-XII: This data window holds the frequency $f_{2}$, sensor fault and the process change that commenced in Window-X.

13) Window-XIII: This data window holds the fault-induced frequencies $f_{1}$ and $f_{2}$, sensor fault, and the process change that commenced in Window-X.

Signals of all the 9 ion chambers are shown in Fig. 6, in which the signals can be seen to have been contaminated by the faults as per the nature of data windows described above.

\section{A. Model development}

The decomposition depth, $J$, to be used for wavelet decomposition can be computed as explained in Sec.III-A from the data of one of the ion chamber signals. Signal data of ion chamber-1 available in window I is chosen for this purpose. From the Fourier transform of this data shown in Fig. 7, it is evident that the magnitude of the transform approaches the noise floor level approximately after $\pi / 64 \mathrm{rad} / \mathrm{sample}$. Thus, the maximum scale for decomposition is selected such that $\pi / 2^{J} \geq \pi / 64$, i.e., $J=6$. Accordingly, the wavelet transformation matrix $\boldsymbol{W}$ for $J=6$ is used in the analysis.

Significant scales can be found out by analysing the wavelet coefficients. From Fig. 8 that shows the wavelet coefficients of signals from ion chamber-1 in Window I, it can be clearly observed that scales 1 and 2 do not carry much information as compared to scales 3 to 6 . A quantitative analysis examining the energy of wavelet coefficients can give further information about information present in different scales. The fraction of energy of detail coefficients from all ion chambers at different scales can be seen in Fig. 9. Both of these preliminary analyses suggest that scales 3 to 6 contain most of the energy for all ion chamber signals as compared to that of scales 1 and 2 . Thus, detail coefficients at scales 1 and 2 can be ignored during reconstruction. Therefore, signal reconstruction is performed by considering detail coefficients from scales 3 to 6 and approximation at scale 6 . The removal of inconsequential detail coefficients is justified, as there is no meaningful loss of useful information in the denoised signal.
Singular value decomposition is performed on different data, such as approximations, details, and reconstructed data, as a first step of PCA. The orders of the PCA models, $m_{j}, j=0,1, \ldots, J+1$, where $j=0$ represents the index of the reconstructed data, while the rest have their usual meaning, are to be chosen based on the CPV curves of their respective data. The CPVs of approximations and reconstructed data are greater than $95 \%$ with only one principal component $(p=1)$ such that the order of the residual subspace $m=9-p=8$. However, for detail coefficients, substantial amount of CPVs are obtained with $p=8$, which make the model order $m=9-p=1$. The matrices $\boldsymbol{P}_{j}$ and $\boldsymbol{B}_{j}, j=0,1, \ldots, J+1$, of all the data are formed based on these model orders. The approximation and detail coefficients of ion chamber- 5 for $J=6$ are shown in Fig. 10. Ion chamber-5 is chosen to present some characteristics of MSPCA-based FDI as the signal corresponding to this sensor is additionally contaminated by a sensor fault from instant 3000 . Since the window I is meant especially for model development, the analysis from the window II onward is presented in the following subsections.

\section{B. No fault signatures in the data}

Approximation and detail coefficients for window II are shown in Fig. 10. The approximation coefficients carry the trend of the data while detail coefficients contain mainly the contribution from noise. This behaviour of MSPCA-based FDI is expected as the ion chamber data carries noisy steady state measurement without any fault signatures or process change in this window.

\section{Faults with localized time-frequency contents}

In the presence of time-frequency localized contents in the data due to some process faults in the reactor, the scales that are sensitive to these contents show variations in their wavelet coefficients. This section demonstrates the outcomes of MSPCA-based FDI in the presence of two frequency modes in the ion chamber data with $f_{1}=5 \mathrm{~Hz}$ and $f_{2}=11 \mathrm{~Hz}$ that correspond to fuel assembly vibrations (refer to Sec. IV) 


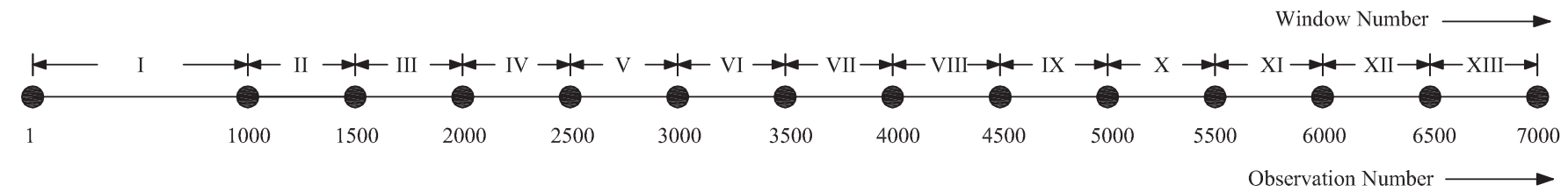

Fig. 4. Data windows for the simulation of different scenarios.

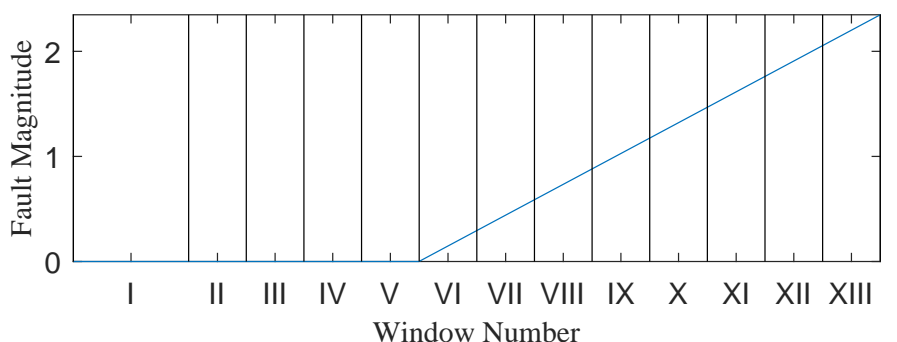

Fig. 5. The growth of the sensor-fault magnitude (mA) in ion chamber-5.

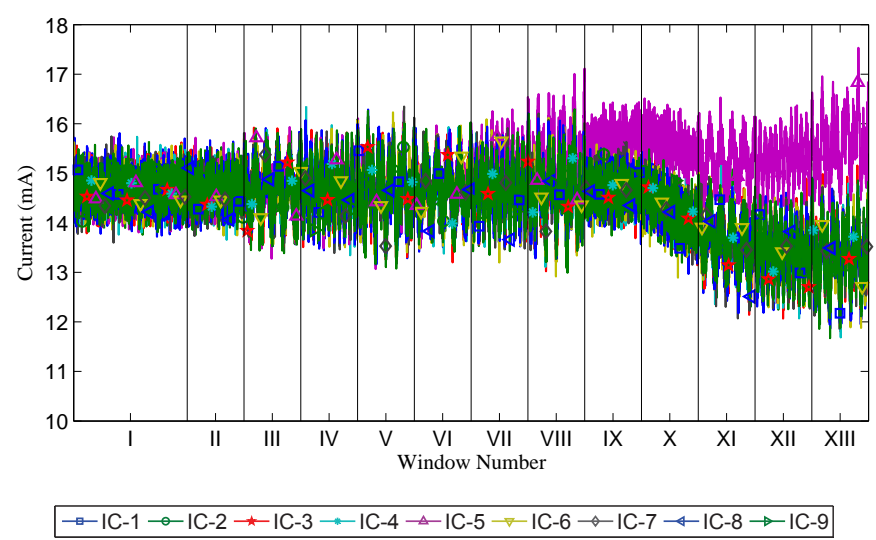

Fig. 6. Signals of all 9 ion chambers.

[23]. These frequency modes are added into the data as per equations (20) and (21) and the data windows presented in Fig. 4.

Fig. 10 shows the approximations and detail coefficients of the data of $5^{\text {th }}$ ion chamber for $J=6$. From Fig. 10 , it can be observed that under the steady-state condition in window II, the approximation coefficients represent slow

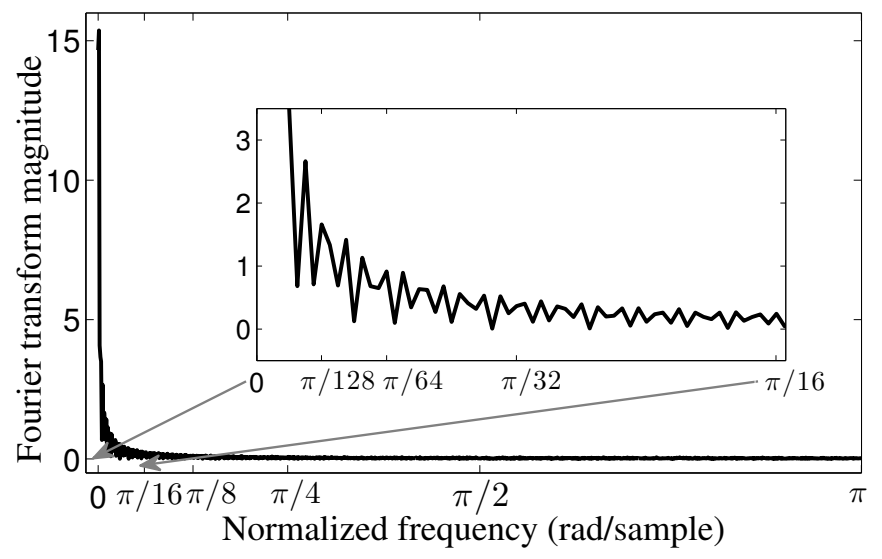

Fig. 7. Fourier transform of signal from first ion chamber.

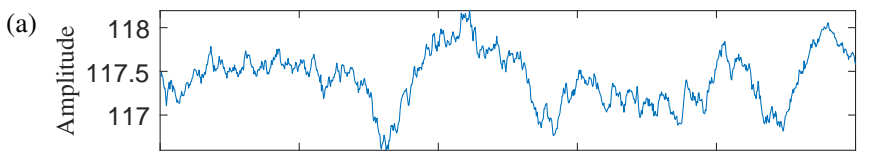

(b)

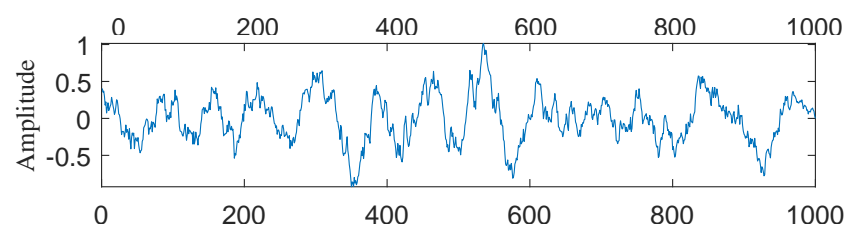

(c)

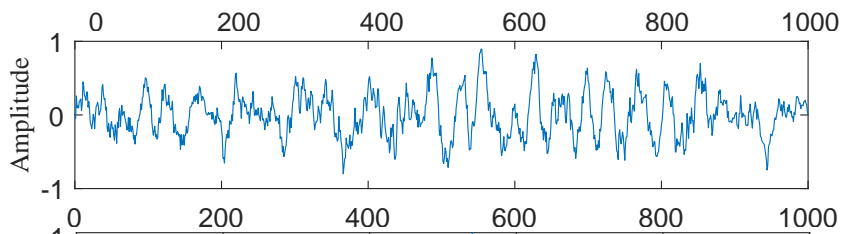

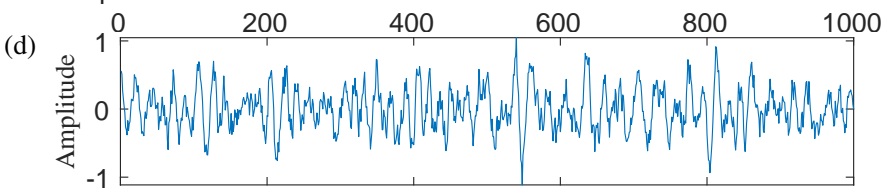
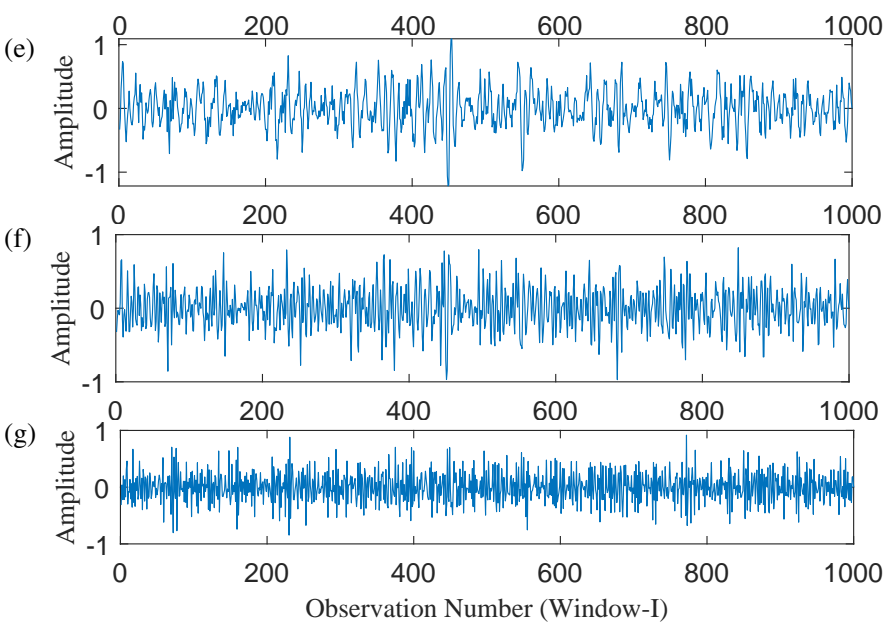

Fig. 8. Fuel assembly vibrations: wavelet coefficients of signal from ion chamber-1 in window I (a) approximations at level-6, (b) details at level-6, (c) details at level-5, (d) details at level-4, (e) details at level-3, (f) details at level-2, and (g) details at level-1.

variation in the process and have non-zero mean whereas the detail coefficients have zero-mean with no auto-correlation. However, the approximations and the detail coefficients have auto-correlated nature with non-zero values when there are process faults accompanied with frequencies either $f_{1}$, or $f_{2}$, or both (windows III to V). The presence of the frequencies $f_{1}=5$ and $f_{2}=11$ can be easily identified respectively in windows III and IV from the approximation and details at level 4 to 6 , while the overlapping of $f_{1}$ on $f_{2}$ can be identified in window V. It can also be observed in Fig. 10 that not all the details coefficients show dynamic variations representative of process faults. To be specific, the sensitivities 


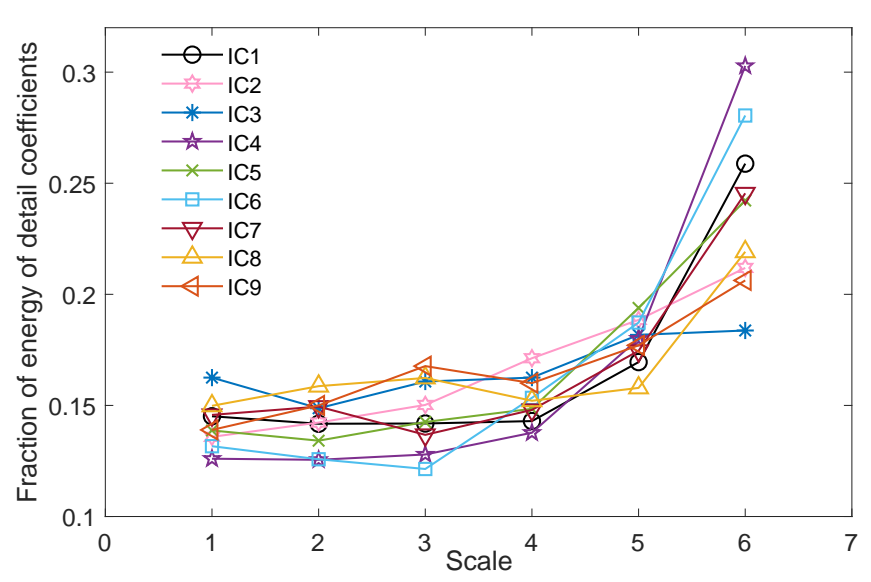

Fig. 9. Fraction of energy of detail coefficients of all ion chamber signals in window I at different scales.

of details at levels 1 and 2 are negligible to the faults with $f_{1}=5 \mathrm{~Hz}$ and $f_{2}=11 \mathrm{~Hz}$, and these details mainly represent the white Gaussian random process. On the other hand, the wavelet coefficients of other ion chambers may exhibit a similar behaviour due to the fact that all ion chambers signals are similarly affected during a process fault.

The ion chamber-5 undergoes a sensor fault from window VI onwards and it can be clearly observed from Fig. 10 by the sudden variation of approximation and details at level 4 to 6 at the onset of window VI. The details at level 1 to 3 can be seen to be insensitive to the sensor fault and they continue to represent the high-frequency components due to process faults. The presence of frequencies in addition to sensor fault in windows VI to VIII, can be detected from the approximation and details coefficients. However, in window IX, no frequency components can be observed from any of these coefficients.

At the commencement of window $\mathrm{X}$, which carries the data of the process change, the approximations drift as they capture the trend of the data, while the details are insensitive to this slowly varying phenomenon. The detection of frequencies in the presence of sensor faults and process change, in windows XI to XIII, can be seen from wavelet coefficients.

Fig. 11 shows the SPE statistics of the wavelet coefficients of all the 9 ion chambers. From Fig. 11(a), it can be observed that the SPE of approximation is insensitive to frequency changes as it is close to zero for the data in windows III to V. The SPEs of details at levels 3 to 6 are fairly above the threshold exactly at the occurrence of frequency modes representative of the faults giving a very good simultaneous time and frequency localization. However, the SPEs obtained for details at levels 1 and 2 do not carry much information. In Fig. 11, the sensitivities of SPEs at levels 3 to 6 are attributed to the changes in the covariance structures of details-data at levels 3 to 6 shown in Fig. 10 from those obtained in the model development. The violation of SPEs above the threshold suggests that there exists a process fault. The detection rate, which is the ratio of the number of fault detection instants and the number of instants at which the fault really exists, is computed at all levels of decomposition in each window for establishing the efficacy of the FDI scheme. Table I gives the detection rates from which the sensitivities of details at scales (a)

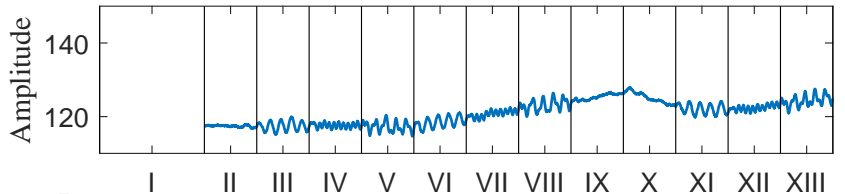

(b)

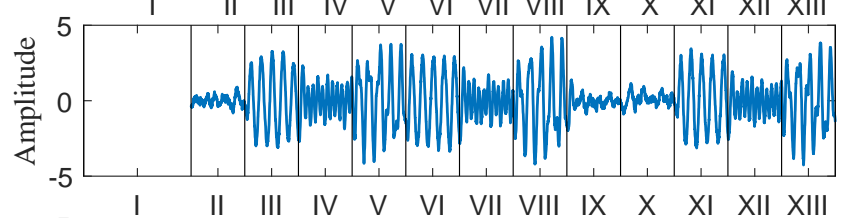

(c)

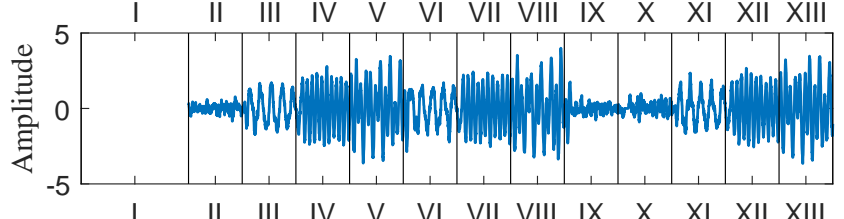

(d)

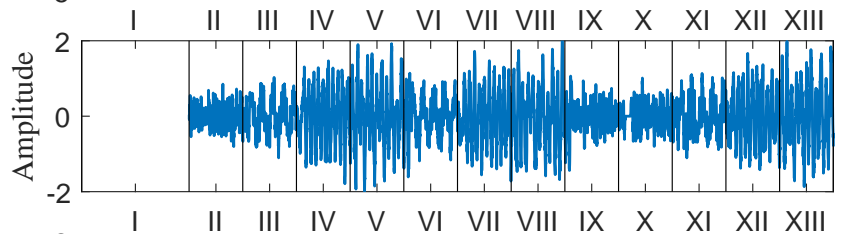

(e)

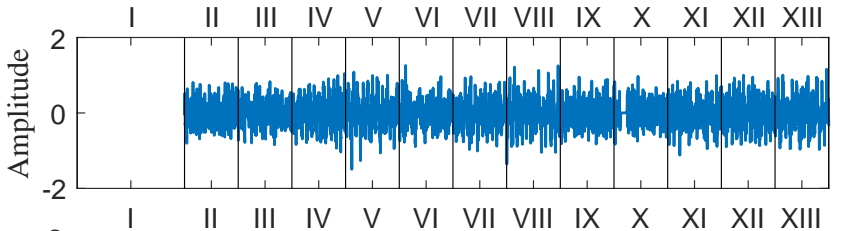

(f)

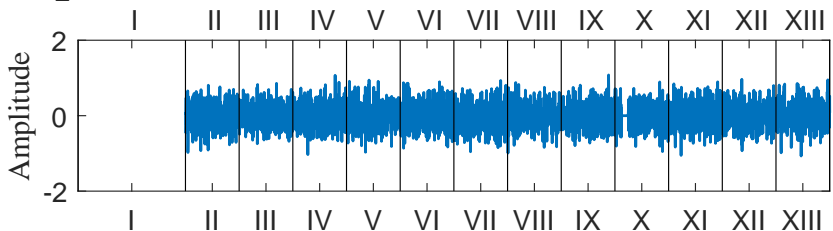

(g)

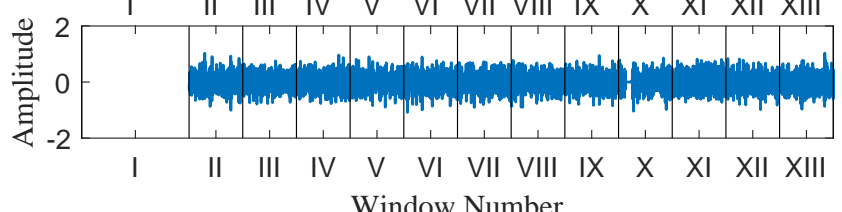

Fig. 10. Fuel assembly vibrations: wavelet coefficients of signal from $5^{\text {th }}$ ion chamber (a) approximations at level-6, (b) details at level-6, (c) details at level-5, (d) details at level-4, (e) details at level-3, (f) details at level-2, and (g) details at level-1.

3 to 6 in response to process faults can be observed.

\section{Sensor fault}

As discussed earlier, there is a sensor fault in the ion chamber-5 from the inception of window VI leading to a change in approximations and details from $k=3000$. SPEs of approximations and details also experience a change at this instant at which the covariance structure of the data is broken. However, SPEs of some finer level details are ignorant of the breakage of covariance structure due to a fault in ion chamber-5. SPEs of the original measurement data before getting fed to the online MSPCA scheme (refer to Fig. $3)$, the reconstructed data obtained with the inverse wavelet transformation and the level- 6 approximations of all 9 ion chambers are respectively shown in Fig. 12(a), 12(b), and Fig. 11(a) along with the thresholds for fault detection. It can be seen from these characteristics that SPEs violate the threshold after $k=3000$ at which the sensor fault arises, such that the 
TABLE I

DETECTION RATES FOR PROCESS FAULTS IN VARIOUS WINDOWS FOR FUEL ASSEMBLY VIBRATIONS.

\begin{tabular}{|c|c|c|c|c|c|c|c|c|c|c|c|c|}
\hline \multirow{2}{*}{ Scale Index } & \multicolumn{12}{|c|}{ Window No. } \\
\hline & II & III & IV & $\mathbf{V}$ & VI & VII & VIII & IX & $\mathbf{X}$ & XI & XII & XIII \\
\hline Approximations at level-6 & 0 & 0 & 0 & 0 & 25.20 & 100 & 100 & 100 & 100 & 100 & 100 & 100 \\
\hline Details at level-6 & 10 & 81.40 & 67.80 & 89 & 85.40 & 66.80 & 82.40 & 28 & 7 & 82.80 & 67.20 & 82.60 \\
\hline Details at level-5 & 4.60 & 79.80 & 91.40 & 89.40 & 83.60 & 89.20 & 90.60 & 15.40 & 7.60 & 78 & 90 & 91.20 \\
\hline Details at level-4 & 2.20 & 5.80 & 14.60 & 15.40 & 4.40 & 7.80 & 17.40 & 11.20 & 1.20 & 5.80 & 12.20 & 17.80 \\
\hline Details at level-3 & 1.20 & 4.80 & 5 & 9.20 & 3 & 5.40 & 9.60 & 5.80 & 1 & 3.20 & 5.60 & 9.20 \\
\hline Details at level-2 & 1 & 1.20 & 1 & 0.40 & 1.40 & 1.20 & 1 & 1.20 & 0.80 & 1 & 1.20 & 1 \\
\hline Details at level-1 & 1.80 & 0.40 & 0.80 & 0.80 & 0.60 & 0.40 & 0.80 & 0.60 & 1 & 1.40 & 1 & 1.20 \\
\hline
\end{tabular}

(a)

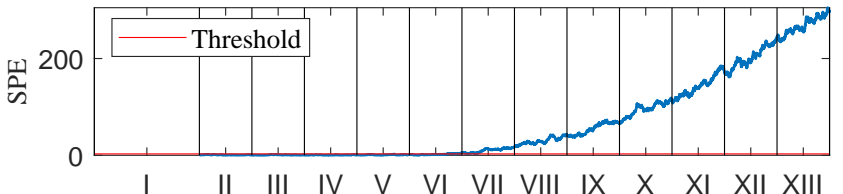

(b)

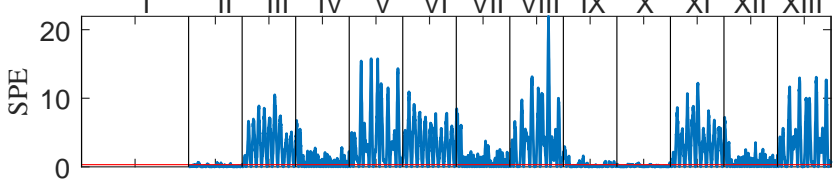

(c)

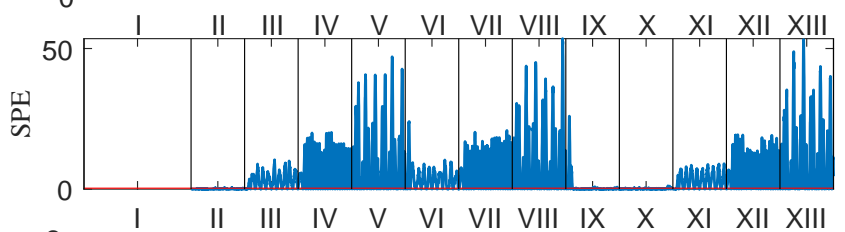

(d)

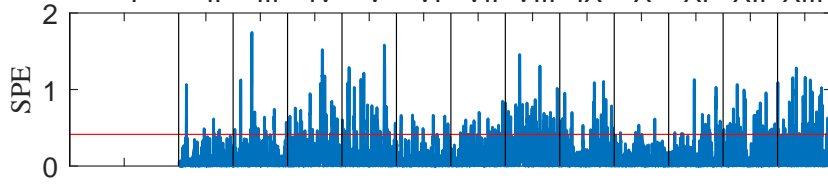

(e)

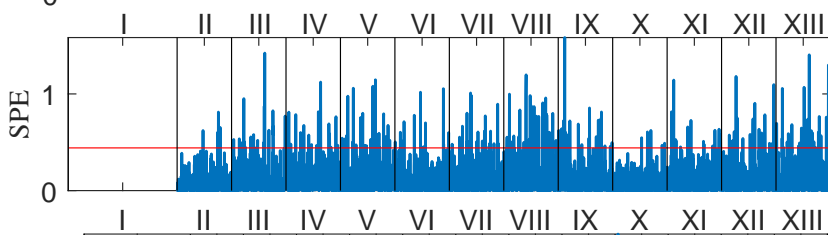

(f)

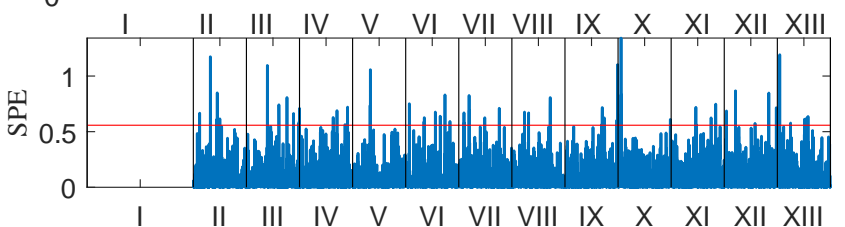

(g)

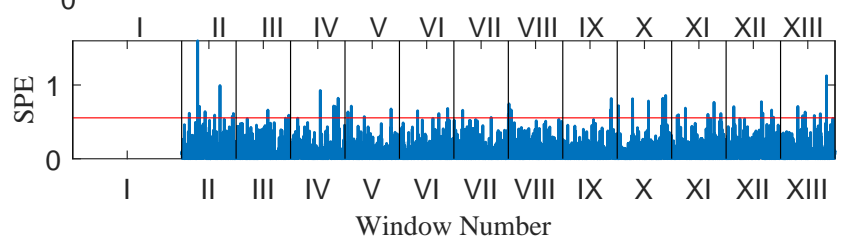

Fig. 11. Fuel assembly vibrations: SPE of (a) approximation coefficients at level-6, (b) detail coefficients at level-6, (c) detail coefficients at level-5, (d) detail coefficients at level-4, (e) detail coefficients at level-3, (f) detail coefficients at level-2, and (g) detail coefficients at level-1. (The horizonta red line represents the threshold for the SPE.)

fault identification (finding of faulty ion chamber) procedure using GLRT is automatically triggered.

Constraint model $(\boldsymbol{A})$ determines the relationship among ion chamber signals and play a crucial role in GLRT. They are obtained through the application of PCA on the abovementioned three variants with an objective of building fault signature vectors. Recall from Section V-A that the CPV calculation led to a model order $m=8$ for these low frequency

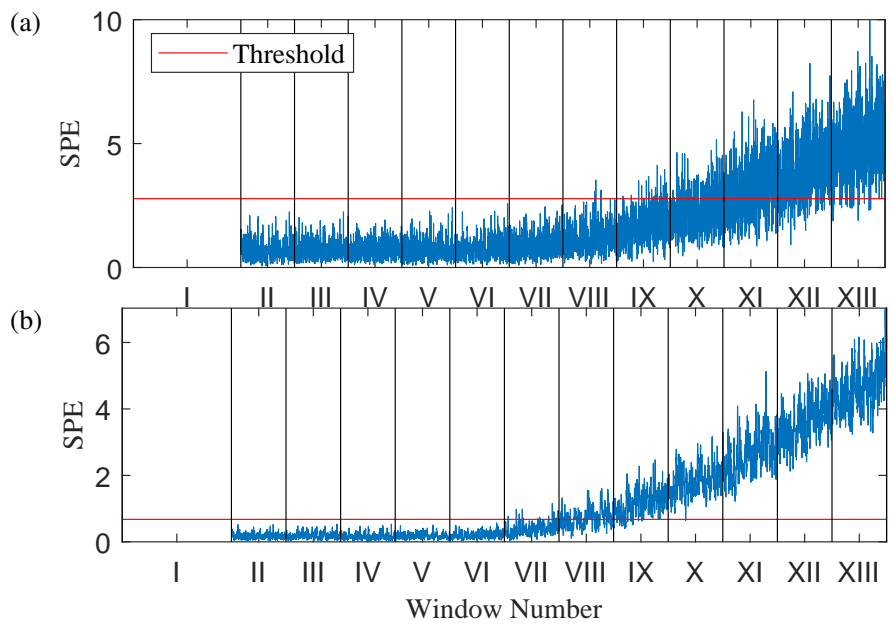

Fig. 12. Sensor Fault Detection: SPEs from (a) the original measurement data, (b) the reconstructed measurement data.

data. Models corresponding to original, reconstructed, and level-6 approximations data are respectively given by $\boldsymbol{A}_{O}$, $\boldsymbol{A}_{R}$ and $\boldsymbol{A}_{A}$ in Appendix-B. It is worth noting that the relationships among ion chamber signals explained by these models obtained from one operating condition of the reactor hold good for other operating conditions including transients also when a successful spatial control scheme is employed [3]

In the simulations, the maximum possible number of simultaneous sensor-faults $g$ is chosen as 1, not only to simplify the demonstration but also due to the fact that more than one ion chamber being simultaneously faulty is unlikely in practice. The fault signature vectors are obtained from the columns of the models (refer to Appendix-B) such that all single-sensorfault scenarios are exhaustively considered as per (10).

GLRT outcomes, namely faulty-sensor index and corresponding fault magnitude ( $\mathrm{mA}$ ), obtained for original, reconstructed, and approximations data are shown respectively in Fig. 13, 14, and 15. On comparing these GLRT outcomes with the corresponding SPEs shown in Fig. 12 and 11(a), it can be observed that there is a set of GLRT outcomes for every SPE violating the threshold. It can be seen that the GLRT analysis based on reconstructed data is better than that based on original data. However, the GLRT applied on approximations data is more prompt in the detection of sensor-faults and more accurate in finding the faulty ion chamber and estimating the corresponding fault magnitude as compared to other two variants. It can also be observed that the fault magnitudes estimated with the GLRT applied on reconstructed data (Fig. 14(b)) and on approximations (Fig. 


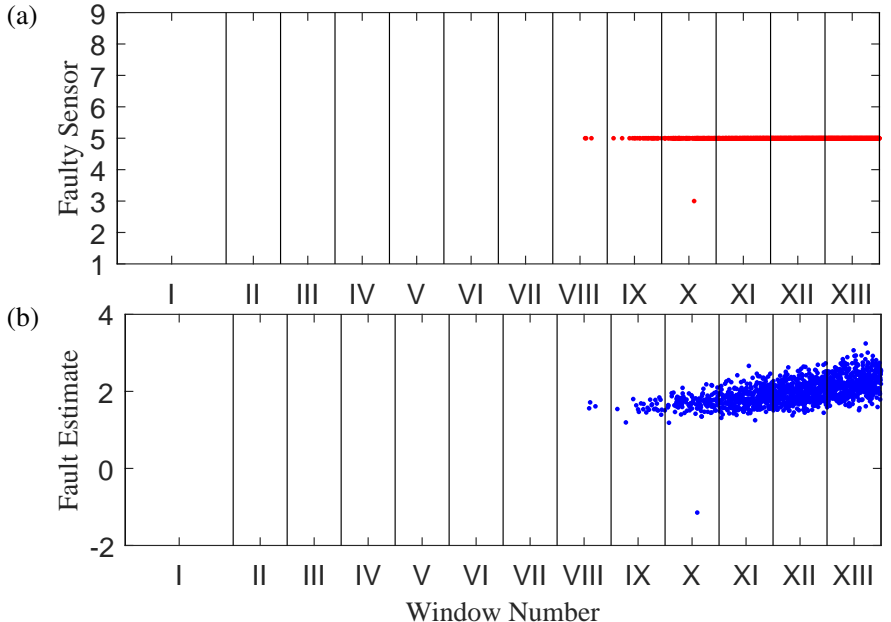

Fig. 13. Sensor-FDI with original measurement data: (a) Faulty sensor index, (b) Estimate of the fault magnitude.

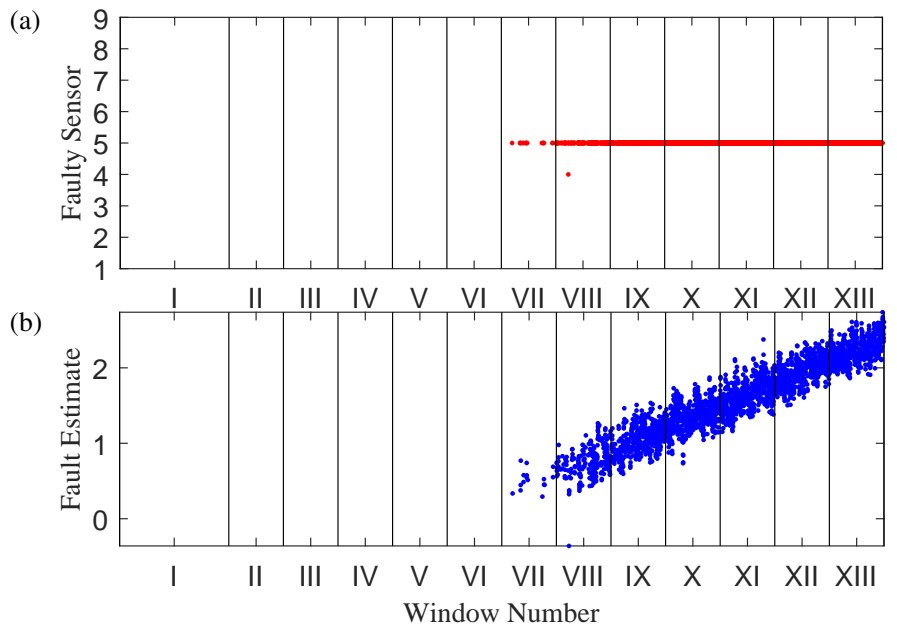

Fig. 14. Sensor-FDI with reconstructed measurement data: (a) Faulty sensor index, (b) Estimate of the fault magnitude.

15(b)) are very much in line with the actual value of fault added in the ion chamber-5 signal. The effectiveness of the FDI with GLRT is expressed in Table II in terms of the indices ODR, OP, and MSE as discussed in Section II-C. It can be seen from Table II that ODR, OP, and MSE obtained from reconstructed data and approximations are better than those obtained with the original data. In particular, the performance based on approximations is very far superior to the other two approaches. From these observations, it can be concluded that the proposed formulation using GLRT is an improved and superior version than the other alternatives. It can also be seen that the proposed scheme yielded consistent results for sensorFDI even during process faults and during a process change.

Summarizing the results obtained in the different simulations, it is possible to state that the MSPCA-based FDI has the ability to handle process faults along with the sensor-FDI and can serve as an important tool for predictive maintenance of reactor internals.

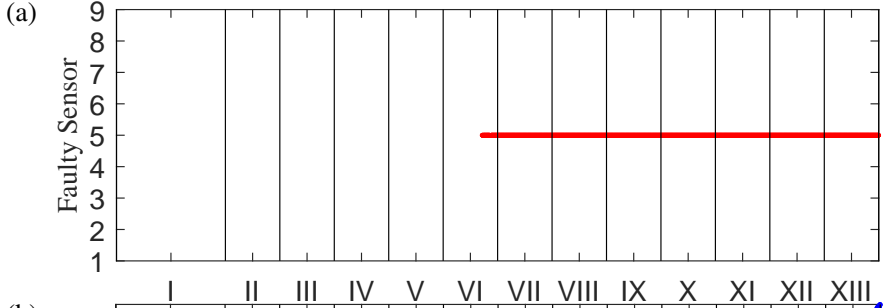

(b)

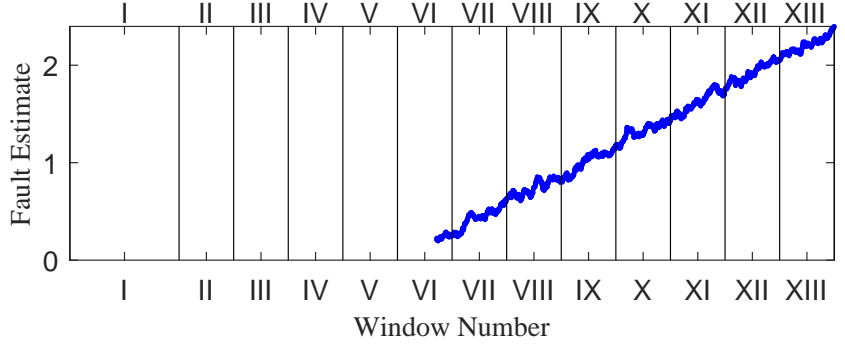

Fig. 15. Sensor-FDI with 6-level approximations data: (a) Faulty sensor index, (b) Estimate of the fault magnitude.

\section{CONCLUSION}

A multi-scale principal component analysis approach for online fault detection and identification in the Advanced Heavy Water Reactor using ex-core ion chambers has been presented. The technique combines proficiency of wavelets in multi-scale data representation and time-frequency localization with fault detection capability of Principal Component Analysis making it well suitable for time-frequency localized faults. Specifically, parsimonious PCA models are developed on significant scales. The squared prediction error statistics are computed at significant scales to detect time-frequency localized events representative of vibration of reactor internals or process faults. Along with the process faults, generalized likelihood ratio test is also applied on original, reconstructed, and approximations with the help of a suitable mathematical formulation to identify the faulty sensor, if any, and the corresponding fault magnitude. Different scenarios have been considered to include various challenges for fault detection and isolation in the reactor operation. Simulation results establish that the proposed scheme is very effective in detecting process faults and sensor faults during both the transient-state as well as the steady-state operation. The results also suggest that it can be a very good tool for predictive maintenance of reactor internals against vibration-induced accidents.

\section{APPENDIX A \\ PRoOF TO LEMMA III.1}

The uni-variate time-series data $\overline{\boldsymbol{y}} \in \mathbb{R}^{2^{J} \times 1}$ in (17) can be expressed as

$$
\overline{\boldsymbol{y}}=\overline{\boldsymbol{x}}+\bar{\varepsilon}+\overline{\boldsymbol{b}},
$$

where $\overline{\boldsymbol{x}}, \overline{\boldsymbol{\varepsilon}}$, and $\overline{\boldsymbol{b}}$ respectively are the true values of the variable being measured, measurement errors and the magnitude of sensor-fault, if any, in the window of $2^{J}$ samples.

From (17) and (18), we have the wavelet approximation coefficients $\boldsymbol{y}_{J+1}$ as the linear combination of $2^{J}$ measurements with the elements in $\tilde{\boldsymbol{H}}_{J}$. Letting $\overline{\boldsymbol{y}}_{J+1}$ be the ordered set of approximation coefficients of all $n$ variables, the covariance 
TABLE II

ODR $(\%)$, OP (\%) AND MSE FOR SENSOR FAULTS IN VARIOUS WINDOWS WITH DIFFERENT DATA VARIANTS

\begin{tabular}{|c|c|c|c|c|c|c|c|c|c|c|c|c|c|}
\hline \multirow{2}{*}{ Data type } & \multirow{2}{*}{ Index } & \multicolumn{12}{|c|}{ Window No. } \\
\hline & & II & III & IV & $\mathbf{V}$ & VI & VII & VIII & IX & $\mathbf{X}$ & XI & XII & XIII \\
\hline \multirow{3}{*}{ Original data } & ODR & 0 & 0 & 0 & 0 & 0 & 0 & 0.60 & 5.20 & 24.80 & 57.80 & 86.00 & 95.80 \\
\hline & $\mathrm{OP}$ & 0 & 0 & 0 & 0 & 0 & 0 & 0.60 & 5.20 & 24.60 & 57.80 & 86 & 95.80 \\
\hline & MSE & - & - & - & - & - & - & 0.85 & 0.52 & 0.41 & 0.31 & 0.26 & 0.2879 \\
\hline \multirow{3}{*}{ Reconstructed data } & ODR & 0 & 0 & 0 & 0 & 0 & 3.20 & 45 & 85.60 & 99.80 & 100 & 100 & 100 \\
\hline & $\mathrm{OP}$ & 0 & 0 & 0 & 0 & 0 & 3.20 & 44.80 & 85.60 & 99.80 & 100 & 100 & 100 \\
\hline & MSE & - & - & - & - & - & 0.15 & 0.18 & 0.16 & 0.16 & 0.17 & 0.15 & 0.15 \\
\hline \multirow{3}{*}{ Approximations data } & ODR & 0 & 0 & 0 & 0 & 25.20 & 100 & 100 & 100 & 100 & 100 & 100 & 100 \\
\hline & $\mathrm{OP}$ & 0 & 0 & 0 & 0 & 25.20 & 100 & 100 & 100 & 100 & 100 & 100 & 100 \\
\hline & MSE & - & - & - & - & 0.02 & 0.04 & 0.04 & 0.04 & 0.04 & 0.03 & 0.03 & 0.02 \\
\hline
\end{tabular}

matrix of measurement error in $\overline{\boldsymbol{y}}_{J+1}$ can be related to that in $\overline{\boldsymbol{y}}$, i.e., $\boldsymbol{\Sigma}_{\varepsilon}$ as

$$
\boldsymbol{\Sigma}_{\varepsilon, J+1}=\tilde{\boldsymbol{H}}_{J} \overline{\boldsymbol{e}} \overline{\boldsymbol{e}}^{T} \tilde{\boldsymbol{H}}_{J}^{T} \boldsymbol{\Sigma}_{\varepsilon}
$$

where $\overline{\boldsymbol{e}} \in \mathbb{R}^{2^{J} \times 1}$ is a column vector with all elements equal to 1 . The covariance matrix of constraint residuals obtained from $\overline{\boldsymbol{y}}_{J+1}$ is [24]

$$
\boldsymbol{\Sigma}_{r, J+1}=\boldsymbol{A}_{A} \boldsymbol{\Sigma}_{\varepsilon, J+1}^{T} \boldsymbol{A}_{A}^{T},
$$

where $\boldsymbol{A}_{A}$ is the PCA-based constraint model obtained from approximation coefficients.

For a hypothesized sensor fault corresponding to $i^{\text {th }}$ variable, the expected value of constraint residuals based on approximation coefficients is

$$
\begin{aligned}
\mathrm{E}[\boldsymbol{r}] & =\mathrm{E}\left[\boldsymbol{A}_{A} \overline{\boldsymbol{y}}_{J+1}\right] \\
& =\boldsymbol{A}_{A} \boldsymbol{E}_{i} \tilde{\boldsymbol{H}}_{J}^{T} \overline{\boldsymbol{b}},
\end{aligned}
$$

where $\boldsymbol{E}_{i} \in \mathbb{R}^{n \times 2^{J}}$ is a matrix whose $i^{\text {th }}$ row has its all elements equal to 1 . Hence, from (A.4), the fault signature matrix in various hypothesized-number of sensor faults up to $g$ is [24]

$\boldsymbol{F}_{i}=\left\{\begin{array}{l}\boldsymbol{A}_{A} \boldsymbol{E}_{i_{1}} \tilde{\boldsymbol{H}}_{J}^{T}, i_{1}=1, \ldots, n ; \\ \boldsymbol{A}_{A}\left(\boldsymbol{E}_{i_{1}} \tilde{\boldsymbol{H}}_{J}^{T}, \boldsymbol{E}_{i_{2}} \tilde{\boldsymbol{H}}_{J}^{T}\right), \forall i_{1}, i_{2}=1, \ldots, n, i_{1} \neq i_{2} ; \ldots ; \\ \boldsymbol{A}_{A}\left(\boldsymbol{E}_{i_{1}} \tilde{\boldsymbol{H}}_{J}^{T}, \boldsymbol{E}_{i_{2}} \tilde{\boldsymbol{H}}_{J}^{T}, \ldots, \boldsymbol{E}_{i_{g}} \tilde{\boldsymbol{H}}_{J}^{T}\right), \\ \quad \forall i_{1}, i_{2}, \ldots, i_{g}=1, \ldots, n, i_{1} \neq i_{2} \neq \ldots \neq i_{g} .\end{array}\right.$

The formulation of GLRT subsequent to this will be on similar lines as that explained in Sec. II-C.

\section{REFERENCES}

[1] H. M. Hashemian, "On-line monitoring applications in nuclear power plants," Prog. Nucl. Energy, vol. 53, no. 2, pp. 167 - 181, 2011.

[2] I. T. Jolliffe, Principal Component Analysis, ser. Springer Series in Statistics. Springer, 2002.

[3] V. S. Yellapu, A. P. Tiwari, and S. B. Degweker, "Application of data reconciliation and fault detection and isolation of ion chambers in Advanced Heavy Water Reactor," Ann. Nucl. Energy, vol. 85, pp. 1210-1225, 2015.

[4] P. Bernard, J. Cloue, C. Messainguiral, R. Baeyens, P. Mathot, J. Satinet, and C. Puyal, "PWR core monitoring by incore noise analysis," Prog. Nucl. Energy, vol. 9, pp. 541 - 556, 1982, reacto Noise - Smorn III.

[5] Y. Fujita and H. Ozaki, "Neutron noise monitoring of reactor core internal vibrations at PWRs in japan," Prog. Nucl. Energy, vol. 9, pp. 423 - 436, 1982, reacto Noise - Smorn III.

[6] O. Glokler, "Reactor noise measurements in the safety and regulating systems of CANDU stations," Prog. Nucl. Energy, vol. 43, no. 1, pp. $75-82,2003$
[7] J. Park, J. H. Lee, T.-R. Kim, J.-B. Park, S. K. Lee, and I.-S. Koo, "Identification of reactor internals' vibration modes of a korean standard PWR using structural modeling and neutron noise analysis," Prog. Nucl. Energy, vol. 43, no. 1, pp. 177 - 186, 2003.

[8] M. Viebach, N. Bernt, C. Lange, D. Hennig, and A. Hurtado, "On the influence of dynamical fuel assembly deflections on the neutron noise level," Prog. Nucl. Energy, vol. 104, pp. 32 - 46, 2018.

[9] M. Mori, M. Kaino, S. Kanemoto, M. Enomoto, S. Ebata, and S. Tsunoyama, "Development of advanced core noise monitoring system for BWRs," Prog. Nucl. Energy, vol. 43, no. 1, pp. 43 - 49, 2003.

[10] A. Kolbasseff and R. Sunder, "Lessons learned with vibration monitoring systems in German nuclear power plants," Prog. Nucl. Energy, vol. 43, no. 1 , pp. $159-165,2003$.

[11] J. Ma and J. Jiang, "Applications of fault detection and diagnosis methods in nuclear power plants: A review," Prog. Nucl. Energy, vol. 53, no. 3, pp. 255 - 266, 2011.

[12] B. R. Upadhyaya and M. Skorska, "Fault analysis of in-core detectors in a PWR using time-series models," in The 22nd IEEE Conference on Decision and Control, 1983, vol. 22, Dec 1983, pp. 758-763.

[13] B. Lu and B. R. Upadhyaya, "Monitoring and fault diagnosis of the steam generator system of a nuclear power plant using data-driven modeling and residual space analysis," Ann. Nucl. Energy, vol. 32, pp. 897-912, 2005.

[14] V. Vajpayee, S. Mukhopadhyay, and A. P. Tiwari, "Multiscale subspace identification of nuclear reactor using wavelet basis function," Ann. Nucl. Energy, vol. 111, pp. $280-292,2018$

[15] C. Shiguo, Z. Ruanyu, W. Peng, and L. Taihua, "Enhance accuracy in pole identification of system by wavelet transform de-noising," IEEE Trans. Nucl. Sci., vol. 51, no. 1, pp. 250-255, Feb 2004.

[16] G. Y. Park, J. Park, and P. Y. Seong, "Application of wavelets noisereduction technique to water-level controller," Nuclear Technology, vol. 145, pp. 177-188, 2004.

[17] B. R. Upadhyaya, C. Mehta, and D. Bayram, "Integration of time series modeling and wavelet transform for monitoring nuclear plant sensors," IEEE Trans. Nucl. Sci., vol. 61, no. 5, pp. 2628-2635, Oct 2014.

[18] E. Nasimi and H. A. Gabbar, "Signal de-noising methods for fault diagnosis and troubleshooting at CANDU ${ }^{\circledR}$ stations," Nucl. Eng. Des., vol. 280, pp. 481-492, 2014

[19] B. R. Bakshi, "Multiscale PCA with application to multivariate statistical process monitoring," AIChE J., vol. 44, no. 7, pp. 1596-1610, 1998

[20] M. Misra, H. Yue, S. Qin, and C. Ling, "Multivariate process monitoring and fault diagnosis by multi-scale pca," Computers \& Chemical Engineering, vol. 26, no. 9, pp. 1281 - 1293, 2002.

[21] S. Yoon and J. F. MacGregor, "Principal-component analysis of multiscale data for process monitoring and fault diagnosis," AIChE Journal, vol. 50, no. 11, pp. 2891-2903, 2004.

[22] R. K. Sinha and A. Kakodkar, "Design and development of the AHWR - the indian thorium fuelled innovative nuclear reactor," Nucl. Eng. and Design, vol. 236, no. 7-8, pp. 683-700, 2006.

[23] "BARC Highlights- AHWR Technology and Engineering." [Online]. Available: https://www.uxc.com/smr/uxc_Library.aspx

[24] S. Narasimhan and C. Jordache, Data Reconciliation \& Gross Error Detection: An Intelligent Use of Process Data. Gulf Publishing, Houston, 2000.

[25] C. Botre, M. Mansouri, M. N. Karim, H. Nounou, and M. Nounou, "Multiscale PLS-based GLRT for fault detection of chemical processes," Journal of Loss Prevention in the Process Industries, vol. 46, pp. 143 153, 2017.

[26] M. Z. Sheriff, M. Mansouri, M. N. Karim, H. Nounou, and M. Nounou, "Fault detection using multiscale PCA-based moving window GLRT," Journal of Process Control, vol. 54, pp. 47 - 64, 2017. 
APPENDIX B

PCA MODELS

\begin{tabular}{|c|c|c|c|c|c|c|c|c|c|}
\hline \multirow{8}{*}{$\boldsymbol{A}_{O} \triangleq \boldsymbol{A}=$} & 0.2891 & 0.6025 & -0.5635 & -0.1708 & 0.2265 & -0.1216 & 0.0185 & -0.3650 & 0.0839 \\
\hline & -0.3957 & -0.0175 & -0.3297 & -0.0014 & -0.0566 & 0.6906 & -0.2872 & 172 & 141 \\
\hline & 0.0104 & -0.3445 & -0.2012 & -0.0169 & 0.3663 & 0.4186 & 0.4903 & 402 & -0.4 \\
\hline & -0.0445 & -0.4117 & -0.1509 & 0.0574 & -0.0150 & -0.3164 & 0.4628 & -0.2384 & 0.6566 \\
\hline & 0.6289 & -0.3173 & 0.1748 & 0.1691 & -0.0443 & 0.1892 & -0.4263 & -0.4658 & 906 \\
\hline & -0.0955 & 0.3225 & 0.3969 & -0.2138 & -0.5417 & 0.2386 & 0.3550 & -0.4552 & -0.0072 \\
\hline & 0.4833 & -0.1702 & -0.2480 & -0.5211 & -0.3729 & 0.1704 & 0.1540 & 0.4589 & 0.0462 \\
\hline & 0.0917 & 0.0610 & -0 & 144 & -0 . & 0.0028 & 0.1423 & 0.0796 & -0 . \\
\hline \multirow{8}{*}{$\boldsymbol{A}_{R} \triangleq \boldsymbol{A}=$} & 0.1296 & -0.1095 & -0.2674 & -0.1158 & 0.4785 & -0.3407 & 0.49 & -0.4 & 0.2274 \\
\hline & -0.0326 & -0.7946 & 0.4231 & 0.1074 & 0.1309 & 0.0213 & 0.2540 & 0.1564 & -0.2655 \\
\hline & 0.5516 & -0.1112 & 0.3238 & 0.3199 & -0.3392 & -0.2447 & -0.2807 & -0.4250 & 0.2048 \\
\hline & -0.1761 & -0.4380 & -0.3197 & 0.1156 & -0.0231 & 0.1754 & -0.2351 & 0.1593 & 0.7414 \\
\hline & 0.1508 & -0.1067 & -0.2579 & 0.0475 & -0.2512 & 0.7600 & 0.2390 & -0.4133 & -0.1688 \\
\hline & -0.6357 & 0.1578 & 0.5321 & -0.0435 & -0.1404 & 0.0875 & 0.1498 & -0.3934 & 0.2854 \\
\hline & 0.0605 & 0.0098 & -0.1069 & -0.0829 & -0.6665 & -0.2576 & 0.5988 & 0.2913 & 0.1545 \\
\hline & -0.3243 & 0.0704 & -0 & 0.8587 & -0. & -0.1 & 0.0939 & -0 . & -0 \\
\hline \multirow{8}{*}{$\boldsymbol{A}_{A} \triangleq \boldsymbol{A}=$} & 0.0124 & 0.0352 & 0.0288 & 0.5114 & -0.4703 & 0.5806 & -0.3359 & -0.1711 & -0.1896 \\
\hline & 0.1932 & 0.1603 & -0.6354 & 0368 & 0.3242 & 0.1254 & -0.26 & -0.3 & 0.4419 \\
\hline & 0.0356 & -0.5018 & 0.3309 & 0.1437 & 0.2973 & 0.0900 & 0.280 & -0.6642 & -0.0133 \\
\hline & -0.0908 & 0.6990 & 0.2722 & -0.4058 & -0.1004 & 0.1041 & 0.0923 & -0.4750 & -0.0965 \\
\hline & 0.7569 & -0.0361 & -0.2869 & -0.1413 & -0.2184 & 0.0100 & 0.3503 & -0.0461 & -0.3884 \\
\hline & -0.2844 & -0.0956 & -0.2359 & -0.3094 & 0.4113 & 0.6164 & 0.1059 & 0.1930 & -0.4012 \\
\hline & 0.1470 & -0.3318 & 0.1697 & -0.5461 & -0.3464 & 0.3663 & -0.06 & $0.0 \xi$ & 0.5256 \\
\hline & -0.4079 & 0.0304 & -0.36 & 0.1648 & -0.3563 & 0.0550 & 0.6918 & -0.0 & 0.2449 \\
\hline
\end{tabular}

[27] D. L. Donoho and I. M. Johnstone, "Adapting to unknown smoothness via wavelet shrinkage," J. American Statis. Assoc., vol. 90, no. 432, pp. 1200-1224, Dec 1995.

[28] S. G. Mallat, A wavelet tour of signal processing: the sparse way, 3rd ed. Academic Press, 2009.

[29] R. A. Razak, M. Bhushan, M. N. Belur, A. P. Tiwari, M. G. Kelkar, and M. Pramanik, "Data reconciliation and gross error analysis of self powered neutron detectors: comparison of PCA and IPCA based models," Int. J. Adv. Eng. Sci. Appl. Math., vol. 4, pp. 91-115, 2012.

[30] M. S. Reis, "A multiscale empirical modeling framework for system identification," Journal of Process Control, vol. 19, no. 9, pp. 15461557, 2009.

[31] V. S. Yellapu, A. P. Tiwari, S. R. Shimjith, M. Naskar, and S. B. Degweker, "Space-time kinetics modeling for the determination of neutron detector signals in Advanced Heavy Water Reactor," in 2013 IEEE International Conference on Control Applications (CCA), Aug 2013, pp. 1224-1229.

[32] V. S. Yellapu, A. K. Mishra, A. P. Tiwari, and S. B. Degweker, "Online fault detection and diagnosis of in-core neutron detectors using generalized likelihood ratio method," IEEE Trans. Nucl. Sci., vol. 62, no. 6, pp. 3311-3323, 2015. 